\title{
Normalisers of operator algebras and tensor product formulas
}

\author{
Martin McGarvey, Lina Oliveira and Ivan G. Todorov
}

\begin{abstract}
We establish a tensor product formula for bimodules over maximal abelian self-adjoint algebras and their supports. We use this formula to show that if $\mathcal{A}$ is the tensor product of finitely many continuous nest algebras, $\mathcal{B}$ is a CSL algebra and $\mathcal{A}$ and $\mathcal{B}$ have the same normaliser semigroup then either $\mathcal{A}=\mathcal{B}$ or $\mathcal{A}^{*}=\mathcal{B}$. We show that the result does not hold without the assumption that the nests be continuous, answering in the negative a question previously raised in the literature.
\end{abstract}

\section{Introduction}

Normalisers of self-adjoint operator algebras were introduced by Murray and von Neumann in the 1930s and have played an important role in operator algebra theory since then. They are used in the study of a number of fundamental classes of operator algebras such as crossed products [30] and limit algebras [23]. Normalisers of tensor products of von Neumann algebras were considered in [4], [12], [26] and [27]. The study of the normalisers of non-self-adjoint operator algebras, namely of nest algebras, was initiated in the 1990s (see [1], [5] and [7]). In [17] the notion of a normaliser was generalised and studied in the context of reflexive algebras, a non-self-adjoint generalisation of von Neumann algebras. It was shown that normalisers are closely related to ternary rings of operators, a class of spaces studied independently in operator space theory (see [3]). This connection provided the basis in [8] for the introduction of an equivalence relation for non-self-adjoint operator algebras, which later lead to the study of a Morita-type equivalence for dual operator algebras [9], [10].

If $\mathcal{A}$ is an operator algebra acting on a Hilbert space $\mathcal{H}$, a normaliser of $\mathcal{A}$ is a bounded linear operator $T$ on $\mathcal{H}$ such that

$$
T^{*} \mathcal{A} T \subseteq \mathcal{A} \text { and } \quad T \mathcal{A} T^{*} \subseteq \mathcal{A} .
$$

Mathematics Subject Classification (2010): Primary 47L35; Secondary 46L10.

Keywords: CSL algebra, masa-bimodule, nest algebra, normaliser. 
Let $N(\mathcal{A})$ be the set of all normalisers of an operator algebra $\mathcal{A}$. It is obvious that $N(\mathcal{A})$ is a self-adjoint semigroup of operators containing the diagonal $\mathcal{A} \cap \mathcal{A}^{*}$ of $\mathcal{A}$ (here and in the sequel we let $\mathcal{A}^{*}=\left\{A^{*}: A \in \mathcal{A}\right\}$ ). The question of to what extent $N(\mathcal{A})$ determines $\mathcal{A}$ was considered in [28], where $\mathcal{A}$ was taken from the class of CSL algebras introduced by Arveson in his seminal work [2]. It is trivial that for any operator algebra $\mathcal{A}$ we have that $N(\mathcal{A})=N\left(\mathcal{A}^{*}\right)$. In the converse direction, easy examples, in which the atomic and the continuous parts of the invariant subspace lattices of the algebras are both nontrivial, show that the equality $N(\mathcal{A})=N(\mathcal{B})$ does not necessarily imply that $\mathcal{B}$ is either equal to $\mathcal{A}$ or to $\mathcal{A}^{*}$. However, it was shown in [28] that $N(\mathcal{A})$ determines $\mathcal{A}$ up to adjoints in the class of totally atomic CSL algebras and in that of continuous nest algebras. The following question was thus raised in [28]: let $\mathcal{A}$ and $\mathcal{B}$ be continuous CSL algebras.

$$
\text { Does } N(\mathcal{A})=N(\mathcal{B}) \text { imply that } \mathcal{A}=\mathcal{B} \text { or } \mathcal{A}^{*}=\mathcal{B} \text { ? }
$$

Within the classes of operator algebras which admit an affirmative answer to the above question, one is able to recover (up to adjoints) non-self-adjoint operator algebras by using self-adjoint objects, namely the normaliser semigroups.

It was shown in [21] that question (1.1) has an affirmative answer if $\mathcal{A}$ is the tensor product of finitely many continuous nest algebras and $\mathcal{B}$ is a CDCSL algebra. In this paper, we extend this result by showing that the answer to question (1.1) is affirmative if $\mathcal{B}$ is any CSL algebra (while $\mathcal{A}$ is still the tensor product of finitely many continuous nest algebras). We show that the assumption on the continuity of the nest algebras cannot be omitted, settling in this way question (1.1) in the negative. After dropping the condition that $\mathcal{B}$ is a CDCSL algebra, we cannot assume the presence of enough Hilbert-Schmidt operators in $\mathcal{B}$, which was the main ingredient in the proofs in [21]. Instead, the main tool we use here is a tensor product formula for bimodules over maximal abelian self-adjoint algebras (masas) which we believe is interesting in its own right.

Tensor product formulas for operator algebras are abundant in the literature. Roughly speaking, given a map $\mathfrak{F}$ defined on a class of operator algebras (or subspaces) closed under tensoring, and taking values in a class of objects, canonically associated with the operator algebras, one asks whether $\mathfrak{F}(\mathcal{A} \otimes \mathcal{B})$ is a suitable kind of a product of $\mathfrak{F}(\mathcal{A})$ and $\mathfrak{F}(\mathcal{B})$. The motivating example is Tomita's commutation theorem where, for a von Neumann algebra $\mathcal{A}$, one sets $\mathfrak{F}(\mathcal{A})$ to be equal to the commutant $\mathcal{A}^{\prime}$ of $\mathcal{A}$. In the case $\mathfrak{F}(\mathcal{A})=\operatorname{Lat} \mathcal{A}$ is the invariant subspace lattice of $\mathcal{A}$, one arrives at the lattice tensor product formula [15], which asks whether $\operatorname{Lat}(\mathcal{A} \otimes \mathcal{B})$ coincides with Lat $\mathcal{A} \otimes \operatorname{Lat} \mathcal{B}$. This formula is known to hold when $\mathcal{A}$ and $\mathcal{B}$ are von Neumann algebras, one of which is injective [24], as well as when $\mathcal{A}$ and $\mathcal{B}$ are Arveson algebras (that is, weak* closed algebras containing a masa) [29]. Weak* closed masa-bimodules, a natural generalisation of Arveson algebras, were examined in [2], [6], [8], [11], [17], and [25]. Their importance is partly due to the fact that they are precisely the weak* closed subspaces invariant under Schur multipliers, a class of maps widely used in various contexts (see e.g. [22]). Here we show that if $\mathcal{U}$ and $\mathcal{V}$ are masa-bimodules then, up to a natural transposition of 
variables, we have that $\operatorname{supp}(\mathcal{U} \otimes \mathcal{V})=\operatorname{supp} \mathcal{U} \times \operatorname{supp} \mathcal{V}$, where $\operatorname{supp} \mathcal{M}$ denotes the support of a masa-bimodule $\mathcal{M}$ introduced in [11] as a subset of the direct product of the coordinate measure spaces corresponding to the two masas. Thus our result can be viewed as a measure-theoretic version of the lattice tensor product formula.

The paper is organised as follows. In Section 2 we collect some definitions and preliminary background. Section 3 is devoted to the tensor product formula for supports. In Section 4 we prove the affirmative result for tensor products of nest algebras stated above, and in Section 5 we give the example showing that the answer to (1.1) is in general negative.

\section{Preliminaries}

All Hilbert spaces in this paper will be assumed to be separable. If $H, H_{1}$ and $H_{2}$ are Hilbert spaces, we denote by $\mathcal{B}\left(H_{1}, H_{2}\right)$ the space of all bounded linear operators from $H_{1}$ into $H_{2}$, and set $\mathcal{B}(H)=\mathcal{B}(H, H)$. Let $(X, \mu)$ and $(Y, \nu)$ be standard measure spaces, that is, the measures $\mu$ and $\nu$ are regular Borel measures with respect to some Borel structures on $X$ and $Y$ arising from complete metrizable topologies. Let $H_{1}=L^{2}(X, \mu), H_{2}=L^{2}(Y, \nu)$, and $\mathcal{D}_{1}$ (respectively, $\mathcal{D}_{2}$ ) be the algebra of all multiplication operators on $H_{1}$ (respectively, $H_{2}$ ) by functions from $L^{\infty}(X, \mu)$ (resp. $L^{\infty}(Y, \nu)$ ). It is well known that $\mathcal{D}_{1}$ and $\mathcal{D}_{2}$ are maximal abelian self-adjoint subalgebras (masas) of $\mathcal{B}\left(H_{1}\right)$ and $\mathcal{B}\left(H_{2}\right)$, respectively. A subspace $\mathcal{U} \subseteq \mathcal{B}\left(H_{1}, H_{2}\right)$ will be called a $\mathcal{D}_{2}, \mathcal{D}_{1}$-bimodule (or a masa-bimodule if $\mathcal{D}_{1}$ and $\mathcal{D}_{2}$ are understood from the context) if $B T A \in \mathcal{U}$ whenever $A \in \mathcal{D}_{1}, B \in \mathcal{D}_{2}$ and $T \in \mathcal{U}$.

We will need several facts and notions from the theory of masa-bimodules (see [2], [11] and [25]). Given a subspace $\mathcal{U} \subseteq \mathcal{B}\left(H_{1}, H_{2}\right)$, we let

$$
\operatorname{Bil} \mathcal{U}=\left\{(P, Q) \in \mathcal{D}_{1} \times \mathcal{D}_{2}: P, Q \text { projections with } Q \mathcal{U} P=\{0\}\right\}
$$

and define, following [20] the reflexive hull of $\mathcal{U}$ by

$$
\operatorname{Ref} \mathcal{U}=\left\{T \in \mathcal{B}\left(H_{1}, H_{2}\right): T \xi \in \overline{\mathcal{U}} \xi \text {, for all } \xi \in H_{1}\right\} .
$$

The space $\mathcal{U}$ is called reflexive $[20]$ if $\mathcal{U}=\operatorname{Ref} \mathcal{U}$.

We equip the direct product $X \times Y$ with the product measure (defined on the $\sigma$ algebra generated by the rectangles $\alpha \times \beta$, where $\alpha \subseteq X$ and $\beta \subseteq Y$ are measurable). A subset $E \subseteq X \times Y$ is called marginally null if $E \subseteq\left(X_{0} \times Y\right) \cup\left(X \times Y_{0}\right)$, where $\mu\left(X_{0}\right)=\nu\left(Y_{0}\right)=0$. We call two subsets $E, F \subseteq X \times Y$ marginally equivalent (and write $E \cong F$ ) if the symmetric difference of $\bar{E}$ and $F$ is marginally null. A set $\kappa \subseteq X \times Y$ is called $\omega$-open if it is marginally equivalent to a (countable) union of the form $\cup_{i=1}^{\infty} \alpha_{i} \times \beta_{i}$, where $\alpha_{i} \subseteq X$ and $\beta_{i} \subseteq Y$ are measurable, $i \in \mathbb{N}$. The complements of $\omega$-open sets are called $\omega$-closed. An operator $T \in \mathcal{B}\left(H_{1}, H_{2}\right)$ is said to be supported on $\kappa$ if, for all rectangles $\alpha \times \beta$ with $(\alpha \times \beta) \cap \kappa \simeq \emptyset$, we have that $P(\beta) T P(\alpha)=0$. (Here $P(\gamma)$ stands for the projection of multiplication by the characteristic function of the measurable subset $\gamma$.) If $\kappa$ is an $\omega$-closed set, let

$$
\mathfrak{M}_{\max }(\kappa)=\left\{T \in \mathcal{B}\left(H_{1}, H_{2}\right): T \text { is supported on } \kappa\right\} .
$$


The space $\mathfrak{M}_{\max }(\kappa)$ is a reflexive masa-bimodule; conversely, every reflexive masa-bimodule is of this form for a unique, up to marginal equivalence, $\omega$-closed set $\kappa,[11]$. Given a masa-bimodule $\mathcal{U}$, its support is the $\omega$-closed set $\kappa$ such that $\operatorname{Ref} \mathcal{U}=\mathfrak{M}_{\max }(\kappa)$.

A subspace lattice on a Hilbert space $\mathcal{H}$ is a lattice of (orthogonal) projections on $\mathcal{H}$ closed in the strong operator topology. Given a subspace lattice $\mathcal{L}$, we let

$$
\operatorname{Alg} \mathcal{L}=\{A \in \mathcal{B}(\mathcal{H}):(I-L) A L=0, \text { for each } L \in \mathcal{L}\}
$$

be the algebra of all operators on $\mathcal{H}$ leaving every projection of $\mathcal{L}$ invariant. Obviously, $\operatorname{Alg} \mathcal{L}$ contains the identity operator, and it is trivial to check that it is closed in the weak operator topology. Conversely, given a unital subalgebra $\mathcal{A} \subseteq \mathcal{B}(\mathcal{H})$, we let

$$
\text { Lat } \mathcal{A}=\{L \in \mathcal{P}(\mathcal{H}):(I-L) A L=0, \text { for each } A \in \mathcal{A}\}
$$

be the lattice of all projections on $\mathcal{H}$ invariant under every operator in $\mathcal{A}$. (Here $\mathcal{P}(\mathcal{H})$ is the lattice of all orthogonal projections on $\mathcal{H}$.) The set $\mathcal{L}$ is easily seen to be a subspace lattice. If $\mathcal{A} \subseteq \mathcal{B}(\mathcal{H})$ is a unital subalgebra then $\operatorname{Ref} \mathcal{A}=\operatorname{Alg} \operatorname{Lat} \mathcal{A}$; thus, $\mathcal{A}$ is reflexive if and only if $\mathcal{A}=\operatorname{Alg} \operatorname{Lat} \mathcal{A}$. By virtue of von Neumann's bicommutant theorem, the class of reflexive algebras contains all von Neumann algebras.

A commutative subspace lattice $(C S L)[2]$ on $\mathcal{H}$ is a subspace lattice $\mathcal{L}$ with the property that $P Q=Q P$ whenever $P, Q \in \mathcal{L}$. An atom of a CSL $\mathcal{L}$ is a non-zero projection $E$ on $\mathcal{H}$ such that for every $L \in \mathcal{L}$, either $E \leq L$ or $E L=0$. A CSL is called continuous if it has no atoms. A CSL algebra is a reflexive algebra $\mathcal{A}$ of the form $\mathcal{A}=\operatorname{Alg} \mathcal{L}$ for some CSL $\mathcal{L}$; equivalently, CSL algebras are the reflexive operator algebras containing a masa.

A nest is a totally ordered CSL, and a nest algebra is an operator algebra $\mathcal{A} \subseteq \mathcal{B}(\mathcal{H})$ of the form $\mathcal{A}=\operatorname{Alg} \mathcal{L}$, for some nest $\mathcal{L}$. If $\mathcal{L}$ is a nest, let $\mathcal{L}^{\perp}=$ $\left\{L^{\bar{\perp}}: L \in \mathcal{L}\right\}$, where for a projection $P$ we have set $P^{\perp}=I-P$; we have that $\operatorname{Alg} \mathcal{L}^{\perp}=(\operatorname{Alg} \mathcal{L})^{*}$.

Throughout the paper, II will denote the interval $[0,1], H$ will denote the Hilbert space $L^{2}(\mathbb{I})$ of square integrable functions on $\mathbb{I}$ equipped with the Lebesgue measure, and $\mathcal{D}$ will denote the multiplication masa of $L^{\infty}(\mathbb{I})$. Let $N_{t}$, for each $t \in \mathbb{I}$, be the projection onto the subspace $\left\{f \in L^{2}(\mathbb{I}): f(s)=0\right.$, for a.e. $\left.s<t\right\}$. The nest $\mathcal{N}_{v}=\left\{N_{t}: 0 \leq t \leq 1\right\}$ is known as the Volterra nest, and the algebra $\mathcal{A}_{v}=\operatorname{Alg} \mathcal{N}_{v}$ is called the Volterra nest algebra. The von Neumann algebra $\mathcal{N}_{v}^{\prime \prime}$ generated by $\mathcal{N}_{v}$ coincides with $\mathcal{D}$ and, in particular, is a masa. Nests with this property are called multiplicity free. (Here, and in the sequel, for a subset $\mathcal{S} \subseteq \mathcal{B}(\mathcal{H})$, we let $\mathcal{S}^{\prime}=\{T \in \mathcal{B}(\mathcal{H}): T S=S T, \forall S \in \mathcal{S}\}$ be the commutant of $\mathcal{S}$ and $\mathcal{S}^{\prime \prime}=\left(\mathcal{S}^{\prime}\right)^{\prime}$ be its second commutant). We refer the reader to [6] for the theory of nest algebras.

If $H_{1}$ and $H_{2}$ are Hilbert spaces, we denote by $H_{1} \otimes H_{2}$ their Hilbertian tensor product. If $\mathcal{A} \subseteq \mathcal{B}\left(H_{1}, \tilde{H}_{1}\right)$ and $\mathcal{B} \subseteq \mathcal{B}\left(H_{2}, \tilde{H}_{2}\right)$ are subspaces, we let $\mathcal{A} \otimes \mathcal{B}$ denote the weak ${ }^{*}$ closed subspace of $\mathcal{B}\left(\hat{H}_{1} \otimes H_{2}, \tilde{H}_{1} \otimes \tilde{H}_{2}\right)$ generated by the elementary tensors $A \otimes B$, where $A \in \mathcal{A}$ and $B \in \mathcal{B}$. Tensor products of nest algebras were studied in detail in [13] where it was shown that if $\mathcal{N}_{i}$ is a nest and $\mathcal{A}_{i}=\operatorname{Alg} \mathcal{N}_{i}$, 
with $i=1, \ldots, n$, then $\mathcal{A}_{1} \otimes \cdots \otimes \mathcal{A}_{n}=\operatorname{Alg}\left(\mathcal{N}_{1} \otimes \cdots \otimes \mathcal{N}_{n}\right)$; here, if $\mathcal{L}_{1}$ and $\mathcal{L}_{2}$ are subspace lattices, $\mathcal{L}_{1} \otimes \mathcal{L}_{2}$ denotes the subspace lattice generated by the projections of the form $P_{1} \otimes P_{2}$, with $P_{1} \in \mathcal{L}_{1}$ and $P_{2} \in \mathcal{L}_{2}$.

\section{The tensor product formula for supports}

In this section, we prove a tensor product formula for supports of masa-bimodules. Let $\left(X_{i}, \mu_{i}\right)$ and $\left(Y_{i}, \nu_{i}\right), i=1,2$, be standard measure spaces, and let $H_{1}=$ $L^{2}\left(X_{1}, \mu_{1}\right), H_{2}=L^{2}\left(X_{2}, \mu_{2}\right), \widetilde{H}_{1}=L^{2}\left(Y_{1}, \nu_{1}\right)$ and $\widetilde{H}_{2}=L^{2}\left(Y_{2}, \nu_{2}\right)$. We also let $\mathcal{D}_{i}$ (respectively, $\widetilde{\mathcal{D}}_{i}$ ) be the multiplication masa of $L^{\infty}\left(X_{i}, \mu_{i}\right)$ (respectively, $\left.L^{\infty}\left(Y_{i}, \nu_{i}\right)\right), i=1,2$. If $\mathcal{U}_{1} \subseteq \mathcal{B}\left(H_{1}, \widetilde{H}_{1}\right)$ is a weak ${ }^{*}$ closed $\widetilde{\mathcal{D}}_{1}, \mathcal{D}_{1}$-bimodule and $\mathcal{U}_{2} \subseteq \mathcal{B}\left(H_{2}, \widetilde{H}_{2}\right)$ is a weak ${ }^{*}$ closed $\widetilde{\mathcal{D}}_{2}, \mathcal{D}_{2}$-bimodule, then $\mathcal{U}_{1} \otimes \mathcal{U}_{2}$ is easily seen to be a $\widetilde{\mathcal{D}}_{1} \otimes \widetilde{\mathcal{D}}_{2}, \mathcal{D}_{1} \otimes \mathcal{D}_{2}$-bimodule. Let

$$
\theta:\left(X_{1} \times X_{2}\right) \times\left(Y_{1} \times Y_{2}\right) \rightarrow\left(X_{1} \times Y_{1}\right) \times\left(X_{2} \times Y_{2}\right)
$$

be the map given by

$$
\theta\left(\left(x_{1}, x_{2}\right),\left(y_{1}, y_{2}\right)\right)=\left(\left(x_{1}, y_{1}\right),\left(x_{2}, y_{2}\right)\right) .
$$

Lemma 3.1. Let $\mathcal{U}_{1} \subseteq \mathcal{B}\left(H_{1}, \widetilde{H}_{1}\right)$ be a $\widetilde{\mathcal{D}}_{1}, \mathcal{D}_{1}$-bimodule and $\mathcal{U}_{2} \subseteq \mathcal{B}\left(H_{2}, \widetilde{H}_{2}\right)$ be a $\widetilde{\mathcal{D}}_{2}, \mathcal{D}_{2}$-bimodule. Then

(i) the support of $\mathcal{U}_{1} \otimes \mathcal{B}\left(H_{2}, \widetilde{H}_{2}\right)$ is marginally equivalent to $\theta^{-1}\left(\operatorname{supp} \mathcal{U}_{1} \times\right.$ $\left.\left(X_{2} \times Y_{2}\right)\right)$, and

(ii) the support of $\mathcal{B}\left(H_{1}, \widetilde{H}_{1}\right) \otimes \mathcal{U}_{2}$ is marginally equivalent to $\theta^{-1}\left(\left(X_{1} \times Y_{1}\right) \times\right.$ $\left.\operatorname{supp} \mathcal{U}_{2}\right)$.

Proof. We only prove (i); part (ii) can be shown similarly. Let $\mathcal{V}=\mathcal{U}_{1} \otimes \mathcal{B}\left(H_{2}, \widetilde{H}_{2}\right)$. Then $\left(\widetilde{\mathcal{D}}_{1} \otimes \mathcal{B}\left(\widetilde{H}_{2}\right)\right) \mathcal{V}\left(\mathcal{D}_{1} \otimes \mathcal{B}\left(H_{2}\right)\right) \subseteq \mathcal{V}$. Let $P \in \mathcal{D}_{1} \otimes \mathcal{D}_{2}$ and $Q \in \widetilde{\mathcal{D}}_{1} \otimes \widetilde{\mathcal{D}}_{2}$ be projections with $Q \mathcal{V} P=\{0\}$. Let $\hat{P}$ be the projection onto the closed hull of $\left(\mathcal{D}_{1} \otimes \mathcal{B}\left(H_{2}\right)\right) P\left(H_{1} \otimes H_{2}\right)$ and $\hat{Q}$ be the projection onto the closed hull of $\left(\widetilde{\mathcal{D}}_{1} \otimes\right.$ $\left.\mathcal{B}\left(\widetilde{H}_{2}\right)\right) Q\left(\widetilde{H}_{1} \otimes \widetilde{H}_{2}\right)$. Fix $\xi \in H_{1} \otimes H_{2}$ and $\eta \in \widetilde{H}_{1} \otimes \widetilde{H}_{2}$. For any $V \in \mathcal{V}$, $C_{1} \in \mathcal{D}_{1} \otimes \mathcal{B}\left(H_{2}\right)$ and $C_{2} \in \widetilde{\mathcal{D}}_{1} \otimes \mathcal{B}\left(\widetilde{H}_{2}\right)$, we have that $C_{2} V C_{1} \in \mathcal{V}$, and therefore $\left\langle V C_{1} P \xi, C_{2}^{*} Q \eta\right\rangle=\left\langle C_{2} V C_{1} P \xi, Q \eta\right\rangle=0$. It follows that $\hat{Q} \mathcal{V} \hat{P}=\{0\}$. Since $\hat{P}$ is invariant under $\mathcal{D}_{1} \otimes \mathcal{B}\left(H_{2}\right)$, we have that $\hat{P}=P_{0} \otimes I$ for some projection $P_{0}$ in $\mathcal{D}_{1}$. Similarly, $\hat{Q}=Q_{0} \otimes I$ for some projection $Q_{0}$ in $\widetilde{\mathcal{D}}_{1}$. It follows that

$$
\left(Q_{0} \mathcal{U}_{1} P_{0}\right) \otimes \mathcal{B}\left(H_{2}, \widetilde{H}_{2}\right)=\{0\}
$$

and, consequently, $Q_{0} \mathcal{U}_{1} P_{0}=\{0\}$; in other words, $\left(P_{0}, Q_{0}\right) \in \operatorname{Bil}_{1}$.

Let $\left\{\left(E_{i}, F_{i}\right)\right\}_{i \in \mathbb{N}}$ be a strongly dense subset of Bil $\mathcal{V}$ and $\alpha_{i} \subseteq X_{1}$ and $\beta_{i} \subseteq Y_{1}$ be measurable sets such that $P\left(\alpha_{i}\right) \otimes I=\hat{E}_{i}$ and $P\left(\beta_{i}\right) \otimes I=\hat{\hat{F}}_{i}$. It follows from the construction given in the proof of Theorem 4.2 in [11] that the support of $\mathcal{V}$ is marginally equivalent to

$$
\left(\bigcup_{i=1}^{\infty}\left(\alpha_{i} \times X_{2}\right) \times\left(\beta_{i} \times Y_{2}\right)\right)^{c} .
$$


On the other hand,

$$
\theta\left(\left(\bigcup_{i=1}^{\infty}\left(\alpha_{i} \times X_{2}\right) \times\left(\beta_{i} \times Y_{2}\right)\right)^{c}\right)=\left(\bigcup_{i=1}^{\infty}\left(\alpha_{i} \times \beta_{i}\right)\right)^{c} \times\left(X_{2} \times Y_{2}\right)
$$

while, as above, $\left(\cup_{i=1}^{\infty}\left(\alpha_{i} \times \beta_{i}\right)\right)^{c}$ is (marginally equivalent to) the support of $\mathcal{U}_{1}$. The proof is complete.

Lemma 3.2. Let $\mathcal{U} \subseteq \mathcal{B}\left(H_{1}, \widetilde{H}_{1}\right)$ be a $\widetilde{\mathcal{D}}_{1}, \mathcal{D}_{1}$-bimodule and let $\mathcal{L}$ be the subspace lattice on $\left(\widetilde{H}_{1} \otimes \widetilde{H}_{2}\right) \oplus\left(H_{1} \otimes H_{2}\right)$ defined by

$$
\mathcal{L}=\left\{\left(\begin{array}{cc}
\widetilde{P}^{\perp} \otimes I & 0 \\
0 & P \otimes I
\end{array}\right):(P, \widetilde{P}) \in \operatorname{Bil} \mathcal{U}\right\} .
$$

Then

$$
\operatorname{Alg} \mathcal{L}=\left(\begin{array}{cc}
\widetilde{\mathcal{D}}_{1} \otimes \mathcal{B}\left(\widetilde{H}_{2}\right) & (\operatorname{Ref} \mathcal{U}) \otimes \mathcal{B}\left(H_{2}, \widetilde{H}_{2}\right) \\
0 & \mathcal{D}_{1} \otimes \mathcal{B}\left(H_{2}\right)
\end{array}\right)
$$

Proof. Since

$$
\operatorname{Ref} \mathcal{U}=\left\{T \in \mathcal{B}\left(H_{1}, \widetilde{H}_{1}\right): Q T P=0, \text { for all }(P, Q) \in \operatorname{Bil} \mathcal{U}\right\},
$$

the right-hand side of the desired identity is trivially contained in its left-hand side. Fix $T=\left(\begin{array}{cc}A & B \\ C & D\end{array}\right) \in \operatorname{Alg} \mathcal{L}$. Then

$$
\left(\begin{array}{cc}
\widetilde{P} \otimes I & 0 \\
0 & P^{\perp} \otimes I
\end{array}\right)\left(\begin{array}{cc}
A & B \\
C & D
\end{array}\right)\left(\begin{array}{cc}
\widetilde{P}^{\perp} \otimes I & 0 \\
0 & P \otimes I
\end{array}\right)=0
$$

for every $(P, \widetilde{P}) \in \operatorname{Bil} \mathcal{U}$. This implies

$$
\begin{aligned}
(\widetilde{P} \otimes I) A\left(\widetilde{P}^{\perp} \otimes I\right) & =0, \\
\left(P^{\perp} \otimes I\right) D(P \otimes I) & =0, \\
\left(P^{\perp} \otimes I\right) C\left(\widetilde{P}^{\perp} \otimes I\right) & =0, \\
(\widetilde{P} \otimes I) B(P \otimes I) & =0
\end{aligned}
$$

for all $(P, \widetilde{P}) \in \operatorname{Bil} \mathcal{U}$. By $(3.1)$, we have that $A \in \widetilde{\mathcal{D}}_{1} \otimes \mathcal{B}\left(\widetilde{H}_{2}\right)$. Similarly, (3.2) implies that $D \in \mathcal{D}_{1} \otimes \mathcal{B}\left(H_{2}\right)$. In (3.3), letting $P=0$ and $\widetilde{P}=0$, we obtain that $C=0$.

Given $\tau \in \mathcal{B}\left(H_{2}, \widetilde{H}_{2}\right)_{*}$, let

$$
L_{\tau}: \mathcal{B}\left(H_{1} \otimes H_{2}, \widetilde{H}_{1} \otimes \widetilde{H}_{2}\right) \rightarrow \mathcal{B}\left(H_{1}, \widetilde{H}_{1}\right)
$$

be the Tomiyama left slice map, that is, the (unique) weak* continuous bounded linear map such that $L_{\tau}(A \otimes B)=\tau(B) A$ for all $A \in \mathcal{B}\left(H_{1}, \widetilde{H}_{1}\right)$ and $B \in \mathcal{B}\left(H_{2}, \widetilde{H}_{2}\right)$. Identity (3.4) implies that $L_{\tau}((\widetilde{P} \otimes I) B(P \otimes I))=0$ for all $\tau \in \mathcal{B}\left(H_{2}, \widetilde{H}_{2}\right)_{*}$ and all $(P, \widetilde{P}) \in \operatorname{Bil} \mathcal{U}$. It easily follows that $\widetilde{P} L_{\tau}(B) P=0$ for all $\tau \in \mathcal{B}\left(H_{2}, \widetilde{H}_{2}\right)_{*}$ and all $(P, \widetilde{P}) \in \operatorname{Bil} \mathcal{U}$ (see (1.3) in [18]). Therefore, $L_{\tau}(B) \in \operatorname{Ref} \mathcal{U}$ for every $\tau \in \mathcal{B}\left(H_{2}, \widetilde{H}_{2}\right)_{*}$. It follows from a subspace version of Theorem 1.9 in [18] that $B \in \operatorname{Ref} \mathcal{U} \otimes \mathcal{B}\left(H_{2}, \widetilde{H}_{2}\right)$, and the proof is complete. 
Lemma 3.3. Let $\mathcal{H}$ be a Hilbert space, let $\mathcal{A} \subseteq \mathcal{B}(\mathcal{H})$ be a weak ${ }^{*}$ closed algebra containing a masa, let $\mathcal{L}=\operatorname{Lat} \mathcal{A}$, and let $E$ be a projection that commutes with $\mathcal{L}$. Then Lat $\left(\left.E \mathcal{A}\right|_{E \mathcal{H}}\right)=\left.\mathcal{L}\right|_{E \mathcal{H}}$.

Proof. Set $\mathcal{A}_{E}=\left.E \mathcal{A}\right|_{E \mathcal{H}}, \mathcal{B}=\operatorname{Ref} \mathcal{A}, \mathcal{B}_{E}=\left.E \mathcal{B}\right|_{E \mathcal{H}}$ and $\mathcal{L}_{E}=\left.\mathcal{L}\right|_{E \mathcal{H}}$. We first prove that $\operatorname{Ref} \mathcal{A}_{E}=\mathcal{B}_{E}$. Note that, by Lemma 1.1 (i) in [28],

$$
\mathcal{B}_{E}=\operatorname{Alg} \mathcal{L}_{E}
$$

and hence $\mathcal{B}_{E}$ is a reflexive operator algebra acting on $E \mathcal{H}$. Since $\mathcal{A}_{E} \subseteq \mathcal{B}_{E}$, we have that Ref $\mathcal{A}_{E} \subseteq \mathcal{B}_{E}$. Suppose that $T \in \mathcal{B}_{E}$. Then $T=\left.E S\right|_{E \mathcal{H}}$ for some $S \in \mathcal{B}$. Since $\mathcal{B}=\operatorname{Ref} \mathcal{A}$, we have that $S x \in \overline{\mathcal{A} x}$, for every $x \in \mathcal{H}$. It follows that, for every $x \in E \mathcal{H}$, the image $E S x$ lies in $\overline{E \mathcal{A} x}$ and, therefore, $T \in \operatorname{Ref} \mathcal{A}_{E}$. Hence $\mathcal{B}_{E} \subseteq \operatorname{Ref} \mathcal{A}_{E}$ and, thus, Ref $\mathcal{A}_{E}$ coincides with $\mathcal{B}_{E}$.

We claim that $\mathcal{L}_{E}$ is a CSL; since it is clearly a lattice of pairwise commuting projections, we need only to show that $\mathcal{L}_{E}$ is closed in the strong operator topology. Suppose that $\left(L_{i}\right)_{i \in \mathbb{N}} \subseteq \mathcal{L}$ is a sequence such that $L_{i} E \rightarrow Q$ for some projection $Q$. Let $\left(L_{i_{k}}\right)_{k \in \mathbb{N}}$ be a subsequence such that $L_{i_{k}} \rightarrow T$ weakly, for some operator $T$. Then $\left(L_{i_{k}}\right)_{k \in \mathbb{N}}$ converges semi-strongly to the projection $M$ onto the kernel of the operator $I-T$. (We refer the reader to [14] for the definition of semi-strong convergence and for the result we use; this notion will not be used in the rest of the paper.) It follows from the definition of semi-strong convergence that $L_{i_{k}} E \rightarrow M E$ semi-strongly. On the other hand, by Proposition 3.1 in [24], $M \in \mathcal{L}$. Since strong convergence implies semi-strong convergence, [14], we have that $Q=M E \in \mathcal{L}_{E}$.

We have showed that $\mathcal{L}_{E}$ is a CSL; by Arveson's theorem [2], it is reflexive. By $(3.5), \mathcal{L}_{E}=\operatorname{Lat} \mathcal{B}_{E}$. By the previous paragraph, Alg Lat $\mathcal{A}_{E}=\mathcal{B}_{E}$ and this implies that

$$
\text { Lat } \mathcal{A}_{E}=\operatorname{Lat} \operatorname{Alg} \text { Lat } \mathcal{A}_{E}=\operatorname{Lat} \mathcal{B}_{E}=\mathcal{L}_{E}
$$

The next theorem is the main result of this section. It can be viewed as a "support" version of the lattice tensor product formula $\operatorname{Lat}(\mathcal{A} \otimes \mathcal{B})=\operatorname{Lat} \mathcal{A} \otimes \operatorname{Lat} \mathcal{B}$, valid for all Arveson algebras [29].

Theorem 3.4. Let $\mathcal{U}_{1} \subseteq \mathcal{B}\left(H_{1}, \widetilde{H}_{1}\right)$ be a $\widetilde{\mathcal{D}}_{1}, \mathcal{D}_{1}$-bimodule and $\mathcal{U}_{2} \subseteq \mathcal{B}\left(H_{2}, \widetilde{H}_{2}\right)$ be a $\widetilde{\mathcal{D}}_{2}, \mathcal{D}_{2}$-bimodule. Then, up to marginal equivalence,

$$
\operatorname{supp}\left(\mathcal{U}_{1} \otimes \mathcal{U}_{2}\right)=\theta^{-1}\left(\operatorname{supp} \mathcal{U}_{1} \times \operatorname{supp} \mathcal{U}_{2}\right) .
$$

Proof. Let $\kappa_{i}=\operatorname{supp} \mathcal{U}_{i}, i=1,2$. Set

$$
\begin{aligned}
& \mathcal{A}_{\mathcal{U}_{1}}=\left\{\left(\begin{array}{cc}
\widetilde{D} & T \\
0 & D
\end{array}\right): D \in \mathcal{D}_{1}, \widetilde{D} \in \widetilde{\mathcal{D}}_{1}, T \in \mathcal{U}_{1}\right\} \subseteq \mathcal{B}\left(\widetilde{H}_{1} \oplus H_{1}\right), \\
& \mathcal{A}_{\mathcal{U}_{2}}=\left\{\left(\begin{array}{cc}
\widetilde{C} & S \\
0 & C
\end{array}\right): C \in \mathcal{D}_{2}, \widetilde{C} \in \widetilde{\mathcal{D}}_{2}, S \in \mathcal{U}_{2}\right\} \subseteq \mathcal{B}\left(\widetilde{H}_{2} \oplus H_{2}\right)
\end{aligned}
$$

and

$$
\mathcal{A}_{\mathcal{U}_{1} \otimes \mathcal{U}_{2}}=\left\{\left(\begin{array}{cc}
\widetilde{E} & R \\
0 & E
\end{array}\right): E \in \mathcal{D}_{1} \otimes \mathcal{D}_{2}, \widetilde{E} \in \widetilde{\mathcal{D}}_{1} \otimes \widetilde{\mathcal{D}}_{2}, R \in \mathcal{U}_{1} \otimes \mathcal{U}_{2}\right\}
$$


so that $\mathcal{A}_{\mathcal{U}_{1} \otimes \mathcal{U}_{2}} \subseteq \mathcal{B}\left(\left(\widetilde{H}_{1} \otimes \widetilde{H}_{2}\right) \oplus\left(H_{1} \otimes H_{2}\right)\right)$. Using (2.1), one can easily check that

$$
\operatorname{Ref} \mathcal{A}_{\mathcal{U}_{1} \otimes \mathcal{U}_{2}}=\left(\begin{array}{cc}
\widetilde{\mathcal{D}}_{1} \otimes \widetilde{\mathcal{D}}_{2} & \operatorname{Ref}\left(\mathcal{U}_{1} \otimes \mathcal{U}_{2}\right) \\
0 & \mathcal{D}_{1} \otimes \mathcal{D}_{2}
\end{array}\right)
$$

The algebra $\mathcal{A}_{\mathcal{U}_{1}} \otimes \mathcal{A}_{\mathcal{U}_{2}}$ acts on the Hilbert space $\mathcal{H}$ defined by

$$
\begin{aligned}
\mathcal{H} & =\left(\widetilde{H}_{1} \oplus H_{1}\right) \otimes\left(\widetilde{H}_{2} \oplus H_{2}\right) \\
& \simeq\left(\widetilde{H}_{1} \otimes \widetilde{H}_{2}\right) \oplus\left(\widetilde{H}_{1} \otimes H_{2}\right) \oplus\left(H_{1} \otimes \widetilde{H}_{2}\right) \oplus\left(H_{1} \otimes H_{2}\right)
\end{aligned}
$$

and, with respect to this decomposition, $\mathcal{A}_{\mathcal{U}_{1}} \otimes \mathcal{A}_{\mathcal{U}_{2}}$ has the matrix form

$$
\left(\begin{array}{cccc}
\widetilde{\mathcal{D}}_{1} \otimes \widetilde{\mathcal{D}}_{2} & \widetilde{\mathcal{D}}_{1} \otimes \mathcal{U}_{2} & \mathcal{U}_{1} \otimes \widetilde{\mathcal{D}}_{2} & \mathcal{U}_{1} \otimes \mathcal{U}_{2} \\
0 & \widetilde{\mathcal{D}}_{1} \otimes \mathcal{D}_{2} & 0 & \mathcal{U}_{1} \otimes \mathcal{D}_{2} \\
0 & 0 & \mathcal{D}_{1} \otimes \widetilde{\mathcal{D}}_{2} & \mathcal{D}_{1} \otimes \mathcal{U}_{2} \\
0 & 0 & 0 & \mathcal{D}_{1} \otimes \mathcal{D}_{2}
\end{array}\right)
$$

Let

$$
E=\left(\begin{array}{cccc}
I & 0 & 0 & 0 \\
0 & 0 & 0 & 0 \\
0 & 0 & 0 & 0 \\
0 & 0 & 0 & I
\end{array}\right) \in \mathcal{B}(\mathcal{H})
$$

Clearly, $E$ belongs to the diagonal of $\mathcal{A}_{\mathcal{U}_{1}} \otimes \mathcal{A}_{\mathcal{U}_{2}}$, and hence commutes with its invariant subspace lattice. Moreover, $\left.E\left(\mathcal{A}_{\mathcal{U}_{1}} \otimes \mathcal{A}_{\mathcal{U}_{2}}\right)\right|_{E \mathcal{H}}=\mathcal{A}_{\mathcal{U}_{1} \otimes \mathcal{U}_{2}}$. It follows from Lemma 3.3 that

$$
\text { Lat } \mathcal{A}_{\mathcal{U}_{1} \otimes \mathcal{U}_{2}}=\left.\left(\operatorname{Lat}\left(\mathcal{A}_{\mathcal{U}_{1}} \otimes \mathcal{A}_{\mathcal{U}_{2}}\right)\right)\right|_{E \mathcal{H}}
$$

By Theorem 4.2 in [29],

$$
\operatorname{Lat}\left(\mathcal{A}_{\mathcal{U}_{1}} \otimes \mathcal{A}_{\mathcal{U}_{2}}\right)=\operatorname{Lat} \mathcal{A}_{\mathcal{U}_{1}} \otimes \text { Lat } \mathcal{A}_{\mathcal{U}_{2}}
$$

It now follows from (3.7) that Lat $\mathcal{A}_{\mathcal{U}_{1} \otimes \mathcal{U}_{2}}$ is generated as a subspace lattice by the projections of the form $\left.(F \otimes G)\right|_{E \mathcal{H}}$, where $F \in$ Lat $\mathcal{A}_{\mathcal{U}_{1}}$ and $G \in \operatorname{Lat} \mathcal{A}_{\mathcal{U}_{2}}$.

If $F \in$ Lat $\mathcal{A}_{\mathcal{U}_{1}}$ and $G \in \operatorname{Lat} \mathcal{A}_{\mathcal{U}_{2}}$ then, by Theorem 3.1 in [25], $F=\left(\begin{array}{cc}\widetilde{P}^{\perp} & 0 \\ 0 & P\end{array}\right)$ and $G=\left(\begin{array}{cc}\widetilde{Q}^{\perp} & 0 \\ 0 & Q\end{array}\right)$ for some $(P, \widetilde{P}) \in \operatorname{Bil} \mathcal{U}_{1}$ and $(Q, \widetilde{Q}) \in \operatorname{Bil}_{2}$. Thus,

$$
F \otimes G=\left(\begin{array}{cccc}
\widetilde{P}^{\perp} \otimes \widetilde{Q}^{\perp} & 0 & 0 & 0 \\
0 & \widetilde{P}^{\perp} \otimes Q & 0 & 0 \\
0 & 0 & P \otimes \widetilde{Q}^{\perp} & 0 \\
0 & 0 & 0 & P \otimes Q
\end{array}\right)
$$

and $\left.(F \otimes G)\right|_{E \mathcal{H}}=\left(\begin{array}{cc}\widetilde{P}^{\perp} \otimes \widetilde{Q}^{\perp} & 0 \\ 0 & P \otimes Q\end{array}\right)$. It follows that Lat $\mathcal{A}_{\mathcal{U}_{1} \otimes \mathcal{U}_{2}}$ is generated as a subspace lattice by the projections of the form

$$
\left(\begin{array}{cc}
I \otimes \widetilde{Q}^{\perp} & 0 \\
0 & I \otimes Q
\end{array}\right),\left(\begin{array}{cc}
\widetilde{P}^{\perp} \otimes I & 0 \\
0 & P \otimes I
\end{array}\right),
$$


where $(P, \widetilde{P}) \in \operatorname{Bil} \mathcal{U}_{1}$ and $(Q, \widetilde{Q}) \in \operatorname{Bil} \mathcal{U}_{2}$. Thus, $\operatorname{Alg}$ Lat $\mathcal{A}_{\mathcal{U}_{1} \otimes \mathcal{U}_{2}}$ is equal to

$$
\operatorname{Alg}\left\{\left(\begin{array}{cc}
\widetilde{P}^{\perp} \otimes I & 0 \\
0 & P \otimes I
\end{array}\right):(P, \widetilde{P}) \in \operatorname{Bil} \mathcal{U}_{1}\right\} \bigcap \operatorname{Alg}\left\{\left(\begin{array}{cc}
I \otimes \widetilde{Q}^{\perp} & 0 \\
0 & I \otimes Q
\end{array}\right):(Q, \widetilde{Q}) \in \operatorname{Bil}_{2}\right\}
$$

by Lemma 3.2 ,

$$
\operatorname{Alg} \operatorname{Lat} \mathcal{A}_{\mathcal{U}_{1} \otimes \mathcal{U}_{2}}=\left(\begin{array}{cc}
\widetilde{\mathcal{D}}_{1} \otimes \mathcal{B}\left(\widetilde{H}_{2}\right) & \operatorname{Ref} \mathcal{U}_{1} \otimes \mathcal{B}\left(H_{2}, \widetilde{H}_{2}\right) \\
0 & \mathcal{D}_{1} \otimes \mathcal{B}\left(H_{2}\right)
\end{array}\right) \bigcap\left(\begin{array}{c}
\mathcal{B}\left(\widetilde{H}_{1}\right) \otimes \widetilde{\mathcal{D}}_{2} \\
0 \\
\mathcal{B}\left(H_{1}, \widetilde{H}_{1}\right) \otimes \operatorname{Ref} \mathcal{U}_{2} \\
\mathcal{B}\left(H_{1}\right) \otimes \mathcal{D}_{2}
\end{array}\right) .
$$

Now (3.6) implies that

$$
\operatorname{Ref}\left(\mathcal{U}_{1} \otimes \mathcal{U}_{2}\right)=\left(\mathcal{B}\left(H_{1}, \widetilde{H}_{1}\right) \otimes \operatorname{Ref} \mathcal{U}_{2}\right) \cap\left(\operatorname{Ref} \mathcal{U}_{1} \otimes \mathcal{B}\left(H_{2}, \widetilde{H}_{2}\right)\right)
$$

By the definition of the support, we have that $\operatorname{supp}\left(\mathcal{U}_{1} \otimes \mathcal{U}_{2}\right)=\operatorname{supp} \operatorname{Ref}\left(\mathcal{U}_{1} \otimes \mathcal{U}_{2}\right)$. On the other hand, if $\mathcal{M}_{1}$ and $\mathcal{M}_{2}$ are reflexive masa-bimodules, then it is easy to see that $\operatorname{supp}\left(\mathcal{M}_{1} \cap \mathcal{M}_{2}\right)=\operatorname{supp} \mathcal{M}_{1} \cap \operatorname{supp} \mathcal{M}_{2}$. It follows that, up to marginal equivalence,

$$
\operatorname{supp}\left(\mathcal{U}_{1} \otimes \mathcal{U}_{2}\right)=\operatorname{supp}\left(\mathcal{B}\left(H_{1}, \widetilde{H}_{1}\right) \otimes \operatorname{Ref} \mathcal{U}_{2}\right) \cap \operatorname{supp}\left(\operatorname{Ref} \mathcal{U}_{1} \otimes \mathcal{B}\left(H_{2}, \widetilde{H}_{2}\right)\right) .
$$

By Lemma 3.1, $\operatorname{supp}\left(\mathcal{U}_{1} \otimes \mathcal{U}_{2}\right)$ is marginally equivalent to $\theta^{-1}\left(\operatorname{supp} \mathcal{U}_{1} \times \operatorname{supp} \mathcal{U}_{2}\right)$.

Let $\left(X_{i}, \mu_{i}\right)$ and $\left(Y_{i}, \nu_{i}\right), i=1, \ldots, n$ be standard measure spaces. Set $H_{i}=$ $L^{2}\left(X_{i}, \mu_{i}\right)$ and $\widetilde{H}_{i}=L^{2}\left(Y_{i}, \nu_{i}\right), i=1, \ldots, n$, and let $\mathcal{D}_{i}$ and $\widetilde{\mathcal{D}}_{i}$ be the multiplication masas of $L^{\infty}\left(X_{i}, \mu_{i}\right)$ and $L^{\infty}\left(Y_{i}, \nu_{i}\right)$ respectively, for $i=1, \ldots, n$. Let

$$
\rho:\left(X_{1} \times \cdots \times X_{n}\right) \times\left(Y_{1} \times \cdots \times Y_{n}\right) \rightarrow\left(X_{1} \times Y_{1}\right) \times \cdots \times\left(X_{n} \times Y_{n}\right)
$$

be given by

$$
\rho\left(\left(x_{1}, x_{2}, \ldots, x_{n}, y_{1}, y_{2}, \ldots, y_{n}\right)\right)=\left(x_{1}, y_{1}, x_{2}, y_{2}, \ldots, x_{n}, y_{n}\right) .
$$

An inductive application of Theorem 3.4 yields the following corollary.

Corollary 3.5. Let $\mathcal{U}_{i} \subseteq \mathcal{B}\left(H_{i}, \widetilde{H}_{i}\right)$ be a $\widetilde{\mathcal{D}}_{i}, \mathcal{D}_{i}$-bimodule for $i=1, \ldots, n$. Then, up to marginal equivalence,

$$
\operatorname{supp}\left(\mathcal{U}_{1} \otimes \cdots \otimes \mathcal{U}_{n}\right)=\rho^{-1}\left(\operatorname{supp} \mathcal{U}_{1} \times \cdots \times \operatorname{supp} \mathcal{U}_{n}\right) .
$$

\section{Tensor products of continuous nest algebras}

Our aim in this section is to establish Theorem 4.11 which shows that tensor products of continuous nest algebras are determined (up to adjoints) by their normaliser semigroups within the class of all CSL algebras. We will need several technical lemmas. 
Let $\mathcal{B}$ be a reflexive operator algebra and let $B$ be an operator in $\mathcal{B}$. The orbit of $B$ with respect to $N(\mathcal{B})$ is the set

$$
\operatorname{Orb}_{N(\mathcal{B})}(B)={\overline{\left[T B T^{*}: T \in N(\mathcal{B})\right.}}^{w *},
$$

where we denote by $[\mathcal{T}]$ the linear span of a subset $\mathcal{T} \subseteq \mathcal{V}$ of a linear space $\mathcal{V}$.

For operator algebras $\mathcal{A}_{i}$, with $i=1, \ldots, n$, let $\mathcal{A}=\mathcal{A}_{1} \otimes \cdots \otimes \mathcal{A}_{n}$ and let $N_{e}(\mathcal{A})=\left\{T_{1} \otimes \cdots \otimes T_{n}: T_{i} \in N\left(\mathcal{A}_{i}\right), i=1, \ldots, n\right\}$. If $P=P_{1} \otimes \cdots \otimes P_{n}$, where for all $i=1, \ldots, n, P_{i}$ is a projection in Lat $\mathcal{A}_{i}$, then let

$$
\begin{array}{r}
N_{P}(\mathcal{A})=\left\{\left(V_{1} \otimes \cdots \otimes V_{n}\right)+\left(W_{1} \otimes \cdots \otimes W_{n}\right): V_{i}, W_{i} \in N\left(\mathcal{A}_{i}\right),\right. \\
\left.V_{i}=P_{i} V_{i} P_{i}, W_{i}=P_{i}^{\perp} W_{i} P_{i}^{\perp}, \forall i=1, \ldots, n\right\} .
\end{array}
$$

The following two lemmas were established in [21].

Lemma 4.1. Let $H_{i}$ be a Hilbert space, let $\mathcal{N}_{i}$ be a continuous nest on $H_{i}$ and let $\mathcal{A}_{i}=\operatorname{Alg} \mathcal{N}_{i}$, with $i=1, \ldots, n$. Let $\mathcal{H}=H_{1} \otimes \cdots \otimes H_{n}$ and let $\mathcal{A}=\mathcal{A}_{1} \otimes \cdots \otimes \mathcal{A}_{n}$. Then the linear span $\left[N_{e}(\mathcal{A})\right]$ of $N_{e}(\mathcal{A})$ is weak* dense in $\mathcal{B}(\mathcal{H})$.

Lemma 4.2. Let $H_{i}$ be a Hilbert space, let $\mathcal{A}_{i}$ be a nest algebra acting on $H_{i}$, with $i=1, \ldots, n$, and let $\mathcal{A}=\mathcal{A}_{1} \otimes \cdots \otimes \mathcal{A}_{n}$. For all $i=1, \ldots, n$, let $\mathcal{C}_{i}$ be either equal to $\mathcal{A}_{i}$ or to $\mathcal{A}_{i}^{*}$, let $\mathcal{C}=\mathcal{C}_{1} \otimes \cdots \otimes \mathcal{C}_{n}$, let $P_{i}$ be a projection in Lat $\mathcal{C}_{i}$ and let $P=P_{1} \otimes \cdots \otimes P_{n}$. Then $N_{P}(\mathcal{C}) \subseteq N(\mathcal{A})$.

It is easy to show that Lemma 4.1 implies the following fact, whose proof we omit.

Lemma 4.3. Let $H_{i}$ be a Hilbert space, let $\mathcal{N}_{i}$ be a continuous multiplicity free nest on $H_{i}$ and let $\mathcal{A}_{i}=\operatorname{Alg} \mathcal{N}_{i}$, with $i=1, \ldots, n$. Let $\mathcal{H}=H_{1} \otimes \cdots \otimes H_{n}$, let $\mathcal{A}=\mathcal{A}_{1} \otimes \cdots \otimes \mathcal{A}_{n}$ and let $A$ be a non-zero operator in $\mathcal{B}(\mathcal{H})$. Then

$$
{\overline{\left[V A W: V, W \in N_{e}(\mathcal{A})\right]}}^{w^{*}}=\mathcal{B}(\mathcal{H}) .
$$

Let $H=L^{2}(\mathbb{I})$ (where the unit interval $\mathbb{I}$ is equipped with Lebesgue measure) and, for any given positive integer $n$, let $\Delta_{+, n}$ be the set defined by

$$
\Delta_{+, n}=\left\{\left(x_{1}, \ldots, x_{n}, y_{1}, \ldots, y_{n}\right) \in \mathbb{I}^{n} \times \mathbb{I}^{n}: x_{i}<y_{i}, i=1, \ldots, n\right\} .
$$

In the sequel, the set $\Delta_{+, 1}$ will be denoted by $\Delta_{+}$.

Lemma 4.4. Let $\mathcal{H}=\underbrace{H \otimes \cdots \otimes H}_{n}$ and let $\kappa$ be an $\omega$-closed subset of $\mathbb{I}^{n} \times \mathbb{I}^{n}$ such that $\kappa \cap \Delta_{+, n} \nsucceq \emptyset$. Then there exist projections $P_{i}$ lying in the Volterra nest $\mathcal{N}_{v}$, with $i=1, \ldots, n$, and a non-zero operator $A$ supported on the set $\kappa$ such that $A=\left(P_{1} \otimes \cdots \otimes P_{n}\right) A\left(P_{1}^{\perp} \otimes \cdots \otimes P_{n}^{\perp}\right)$. 
Proof. Let $\mathbf{0}$ and $\mathbf{1}$ be the elements of $\mathbb{I}^{n}$ respectively defined by $\mathbf{0}=(0, \ldots, 0)$ and $\mathbf{1}=(1, \ldots, 1)$. For an element $r=\left(r_{1}, \ldots, r_{n}\right)$ in $\mathbb{I}^{n}$, write

$$
[r, \mathbf{1}]=\left\{\left(s_{1}, \ldots, s_{n}\right) \in \mathbb{I}^{n}: r_{i} \leq s_{i} \leq 1, i=1, \ldots, n\right\}
$$

and

$$
[\mathbf{0}, r)=\left\{\left(s_{1}, \ldots, s_{n}\right) \in \mathbb{I}^{n}: 0 \leq s_{i}<r_{i}, i=1, \ldots, n\right\} .
$$

Let $\left\{t_{j}\right\}_{j \in \mathbb{N}}$ be a dense subset of $\mathbb{I}^{n}$ and, for all $j \in \mathbb{N}$, suppose that

$$
\kappa \bigcap\left(\left[\mathbf{0}, t_{j}\right) \times\left[t_{j}, \mathbf{1}\right]\right) \simeq \emptyset .
$$

Since the union of countably many marginally null sets is marginally null, this implies that

$$
\kappa \cap \Delta_{+, n}=\bigcup_{j=1}^{\infty}\left(\kappa \cap\left(\left[\mathbf{0}, t_{j}\right) \times\left[t_{j}, \mathbf{1}\right]\right)\right) \simeq \emptyset,
$$

yielding a contradiction. Hence, $\kappa \cap\left(\left[\mathbf{0}, t_{j}\right) \times\left[t_{j}, \mathbf{1}\right]\right) \not \emptyset$ for some $j \in \mathbb{N}$. Since the set $\kappa \cap\left(\left[\mathbf{0}, t_{j}\right) \times\left[t_{j}, \mathbf{1}\right]\right)$ is $\omega$-closed, Arveson's null set theorem (see Theorem 1.4.3 in [2]) implies that there exists a non-zero operator $A$ supported on $\kappa \cap$ $\left(\left[\mathbf{0}, t_{j}\right) \times\left[t_{j}, \mathbf{1}\right]\right)$. Clearly, $A$ is supported on the set $\kappa$ and $A=P\left(\left[t_{j}, \mathbf{1}\right]\right) A P\left(\left[\mathbf{0}, t_{j}\right]\right)$ The conclusion follows from the fact that $L_{j}=P_{1} \otimes \cdots \otimes P_{n}$ and $M_{j}=P_{1}^{\perp} \otimes \cdots \otimes$ $P_{n}^{\perp}$, where, if $t_{j}=\left(t_{j}^{1}, \ldots, t_{j}^{n}\right)$, we have set $P_{i}=P\left(\left[t_{j}^{i}, 1\right]\right), i=1, \ldots, n$.

Lemma 4.5. Let $E$ and $F$ be projections in the Volterra nest $\mathcal{N}_{v}$ such that $0<$ $E<I$ and $0<F<I$. Then there exists a unitary operator $W$ lying in $N\left(\mathcal{A}_{v}\right)$ with $W E W^{*}=F$.

Proof. Let $s$ and $t$ be real numbers with $0<s, t<1$ and such that $E$ is the projection onto the space $L^{2}(s, 1)$ and $F$ is the projection onto the space $L^{2}(t, 1)$. Let $\mathcal{N}_{E}$ and $\mathcal{N}_{F}$ be the nests defined by $\mathcal{N}_{E}=\left.\mathcal{N}_{v}\right|_{E H}$ and $\mathcal{N}_{F}=\left.\mathcal{N}_{v}\right|_{F H}$. The nests $\mathcal{N}_{E}$ and $\mathcal{N}_{F}$ are continuous and multiplicity free; it follows that there exists a unitary operator $U: L^{2}(s, 1) \rightarrow L^{2}(t, 1)$ such that $U \mathcal{N}_{E} U^{*}=\mathcal{N}_{F}$ (see [6]). After identifying $U$ with the operator $U \oplus 0$ acting on $H$, we have that $U E U^{*}=F$.

Similarly, the nests $\mathcal{N}_{E \perp}$ and $\mathcal{N}_{F \perp}$, defined by $\mathcal{N}_{E^{\perp}}=\left.\mathcal{N}_{v}\right|_{E^{\perp} H}$ and $\mathcal{N}_{F^{\perp}}=$ $\left.\mathcal{N}_{v}\right|_{F^{\perp} H}$, are unitarily equivalent. Hence there exists a unitary operator $V$ : $L^{2}(0, s) \rightarrow L^{2}(0, t)$ such that $V \mathcal{N}_{E^{\perp}} V^{*}=\mathcal{N}_{F^{\perp}}$. After identifying $V$ with the operator $0 \oplus V$ acting on $H$, we have that $V E^{\perp} V^{*}=F^{\perp}$.

It is clear that the operator $W$, defined by $W=U+V$, is a unitary operator on $H$ and that $W E W^{*}=U E U^{*}=F$. Let $A$ be an operator in the Volterra nest algebra $\mathcal{A}_{v}$. Then

$$
W A W^{*}=U A U^{*}+U A V^{*}+V A U^{*}+V A V^{*}
$$

and, since $U, V \in N\left(\mathcal{A}_{v}\right)$, we have that $U A U^{*}, V A V^{*} \in \mathcal{A}_{v}$. A direct verification shows that $V A U^{*}=0$.

If $L$ is a projection lying in $\mathcal{N}_{v}$, then there exists $w \in[0,1]$ such that $L H=$ $L^{2}(w, 1)$. Since the initial space of $V^{*}$ is $L^{2}(0, t)$, if there exists $\xi \in L H$ with $V^{*} \xi \neq 0$, 
then the inequality $w<t$ must hold. Therefore $L^{2}(t, 1)$ is a subspace of $L H$, yielding that

$$
U A V^{*} \xi \in L^{2}(t, 1) \subseteq L H .
$$

Hence the operator $U A V^{*}$ lies in $\mathcal{A}_{v}$, and it follows that $W$ is a normaliser of $\mathcal{A}_{v}$.

The next theorem shows that the normalisers of the tensor product of finitely many copies of the Volterra nest algebra act "transitively" on certain non-zero operators.

Theorem 4.6. Let $H_{i}$ be a Hilbert space, let $\mathcal{N}_{i}$ be a continuous multiplicity free nest acting on $H_{i}$ and let $\mathcal{A}_{i}=\operatorname{Alg} \mathcal{N}_{i}$, with $i=1, \ldots, n$. Let $\mathcal{H}=H_{1} \otimes \cdots \otimes H_{n}$ and let $\mathcal{A}=\mathcal{A}_{1} \otimes \cdots \otimes \mathcal{A}_{n}$. Let $A$ be a non-zero operator lying in $\mathcal{A}$ such that $A=\left(P_{1} \otimes \cdots \otimes P_{n}\right) A\left(P_{1}^{\perp} \otimes \cdots \otimes P_{n}^{\perp}\right)$ for some $P_{i} \in \mathcal{N}_{i}$, with $i=1, \ldots, n$. Then the sets $\overline{\left[(S T) A(S T)^{*}: S \in N_{e}(\mathcal{A}), T \in N_{P}(\mathcal{A})\right]}{ }^{w^{*}}, \mathcal{A}$ and $\operatorname{Orb}_{N(\mathcal{A})}(A)$ coincide.

Proof. In the conditions of the theorem, let $P=P_{1} \otimes \cdots \otimes P_{n}$, let $Q=P_{1}^{\perp} \otimes \cdots \otimes P_{n}^{\perp}$, and, for all $i=1, \ldots, n$, let $V_{i}$ and $W_{i}$ be normalisers of $\mathcal{A}_{i}$ such that $V_{i}=P_{i} V_{i} P_{i}$ and $W_{i}=P_{i}^{\perp} W_{i} P_{i}^{\perp}$. Define the operators $V=V_{1} \otimes \cdots \otimes V_{n}, W=W_{1} \otimes \cdots \otimes W_{n}$ and $T=V+W$. Since $A=P A Q$, we have that

$$
T A T^{*}=(T P) A\left(Q T^{*}\right)=V A W^{*} .
$$

Let $\mathcal{C}_{i}=\left.P_{i} \mathcal{A}_{i}\right|_{P_{i} H_{i}}$ and let $\mathcal{B}_{i}=\left.P_{i}^{\perp} \mathcal{A}_{i}\right|_{P_{i}^{\perp} H_{i}}$, with $i=1, \ldots, n$, and suppose that all operators $S$ (resp. $R$ ) acting on $P_{i} H_{i}$ (resp. $P_{i}^{\perp} H_{i}$ ) are identified with $S \oplus 0$ (resp. $0 \oplus R$ ) acting on $H_{i}$. Since, by Lemma 1.1 (ii) in [28], the equality $N\left(\left.E \mathcal{A}_{i}\right|_{E H_{i}}\right)=\left.E N\left(\mathcal{A}_{i}\right)\right|_{E H_{i}}$ holds for every projection $E \in \mathcal{N}_{i}$, it now follows from (4.2) that

$$
\begin{array}{r}
\left\{T A T^{*}: T \in N_{P}(\mathcal{A})\right\}=\left\{V A W: V=V_{1} \otimes \cdots \otimes V_{n}, W=W_{1} \otimes \cdots \otimes W_{n},\right. \\
\left.V_{i} \in N\left(\mathcal{C}_{i}\right), W_{i} \in N\left(\mathcal{B}_{i}\right), i=1, \ldots, n\right\} .
\end{array}
$$

The algebras $\mathcal{C}_{i}$ and $\mathcal{B}_{i}$ are continuous multiplicity free nest algebras, and hence each one of them is unitarily equivalent to $\mathcal{A}_{v}$. Lemma 4.3 now implies that

$$
P \mathcal{B}(\mathcal{H}) Q \subseteq{\overline{\left[T A T^{*}: T \in N_{P}(\mathcal{A})\right.}}^{w^{*}} .
$$

For all $i=1, \ldots, n$, let $E_{i}$ be a projection in $\mathcal{N}_{i}$ satisfying $0<E_{i}<I$. By Lemma 4.5 , for all $i=1, \ldots, n$, there exists a unitary operator $U_{i} \in N\left(\mathcal{A}_{i}\right)$ such that $U_{i} P_{i} U_{i}^{*}=E_{i}$. Thus, if we define the operators $E=E_{1} \otimes \cdots \otimes E_{n}$, $F=E_{1}^{\perp} \otimes \cdots \otimes E_{n}^{\perp}$ and $U=U_{1} \otimes \cdots \otimes U_{n}$, then $U \in N_{e}(\mathcal{A})$ and $U P \mathcal{B}(\mathcal{H}) Q U^{*}=$ $E \mathcal{B}(\mathcal{H}) F$. Hence,

$$
E \mathcal{B}(\mathcal{H}) F \subseteq \overline{\left[(S T) A(S T)^{*}: S \in N_{e}(\mathcal{A}), T \in N_{P}(\mathcal{A})\right]^{*}} .
$$

It follows from Lemma 3.1 in [21] that the span of the spaces of the form $E \mathcal{B}(\mathcal{H}) F$ is weak* dense in $\mathcal{A}$, and hence

$$
\mathcal{A} \subseteq \overline{\left[(S T) A(S T)^{*}: S \in N_{e}(\mathcal{A}), T \in N_{P}(\mathcal{A})\right]^{w^{*}}} .
$$


By Lemma 4.2, we have that the set on the right-hand side is contained in $\operatorname{Orb}_{N(\mathcal{A})}(A)$. On the other hand, since $A \in \mathcal{A}$, we have that $\operatorname{Orb}_{N(\mathcal{A})}(A) \subseteq \mathcal{A}$, and the proof is complete.

Remark. It can be shown, using similar arguments to the ones given above, that, in the notation of Theorem 4.6, if the operator $A$ lies in the Schatten $\mathcal{C}_{p}$-class, $1 \leq p \leq \infty$, then

$$
{\overline{\left[T A T^{*}: T \in N(\mathcal{A})\right.}}^{\|\cdot\|_{p}}=\mathcal{A} \cap \mathcal{C}_{p}(\mathcal{H}) .
$$

Here, as usual, $\mathcal{C}_{\infty}$ is the ideal of compact operators.

The following lemma can be established easily using elementary slice map techniques, and we omit its proof.

Lemma 4.7. Let $\mathcal{A}_{1}$ and $\mathcal{A}_{2}$ be reflexive operator subalgebras of $\mathcal{B}\left(\mathcal{H}_{1}\right)$ and $\mathcal{B}\left(\mathcal{H}_{2}\right)$, respectively, and let $S \otimes T \in N\left(\mathcal{A}_{1} \otimes \mathcal{A}_{2}\right)$. If $T \neq 0$, then $S \in N\left(\mathcal{A}_{1}\right)$ and if $S \neq 0$, then $T \in N\left(\mathcal{A}_{2}\right)$.

Lemma 4.8. Let $\mathcal{H}_{1}$ and $\mathcal{H}_{2}$ be Hilbert spaces and let $\mathcal{A}_{1}$ and $\mathcal{B}_{1}$ (resp. $\mathcal{A}_{2}$ and $\left.\mathcal{B}_{2}\right)$ be reflexive operator algebras acting on $\mathcal{H}_{1}\left(\right.$ resp. $\left.\mathcal{H}_{2}\right)$. If $N\left(\mathcal{A}_{1} \otimes \mathcal{A}_{2}\right)=$ $N\left(\mathcal{B}_{1} \otimes \mathcal{B}_{2}\right)$, then $N\left(\mathcal{A}_{1}\right)=N\left(\mathcal{B}_{1}\right)$ and $N\left(\mathcal{A}_{2}\right)=N\left(\mathcal{B}_{2}\right)$.

Proof. Suppose that $N\left(\mathcal{A}_{1} \otimes \mathcal{A}_{2}\right)$ coincides with $N\left(\mathcal{B}_{1} \otimes \mathcal{B}_{2}\right)$. If $S \in N\left(\mathcal{A}_{1}\right)$, then $S \otimes I \in N\left(\mathcal{A}_{1} \otimes \mathcal{A}_{2}\right)$ and therefore $S \otimes I \in N\left(\mathcal{B}_{1} \otimes \mathcal{B}_{2}\right)$. Hence, by Lemma 4.7, the operator $S$ lies in $N\left(\mathcal{B}_{1}\right)$. It follows that $N\left(\mathcal{A}_{1}\right) \subseteq N\left(\mathcal{B}_{1}\right)$, and, by symmetry, $N\left(\mathcal{A}_{1}\right)=N\left(\mathcal{B}_{1}\right)$. Similarly we can show that $N\left(\mathcal{A}_{2}\right)=N\left(\mathcal{B}_{2}\right)$.

Lemma 4.9. Let $\mathcal{A}=\underbrace{\mathcal{A}_{v} \otimes \cdots \otimes \mathcal{A}_{v}}_{n}$ and let $\mathcal{B}=\mathcal{B}_{1} \otimes \cdots \otimes \mathcal{B}_{n}$, where, for all $i=1, \ldots, n, \mathcal{B}_{i}$ coincides either with $\mathcal{A}_{v}$ or with $\mathcal{A}_{v}^{*}$. Then $N(\mathcal{A})=N(\mathcal{B})$ if and only if $\mathcal{A}=\mathcal{B}$ or $\mathcal{A}^{*}=\mathcal{B}$.

Proof. First, suppose that $n=2$ and that $\mathcal{B}=\mathcal{A}_{v} \otimes \mathcal{A}_{v}^{*}$. We show that $N(\mathcal{A}) \neq$ $N(\mathcal{B})$. Let $W$ in $\mathcal{B}(H \otimes H)$ be the unitary operator defined, for all $\xi, \eta \in H$, by $W(\xi \otimes \eta)=\eta \otimes \xi$. If $P_{1}$ and $P_{2}$ are projections lying on $H$, then $W\left(P_{1} H \otimes P_{2} H\right)=$ $P_{2} H \otimes P_{1} H$. Hence, given projections $P_{1}, P_{2} \in \mathcal{N}_{v}$, for all operators $A$ lying in $\mathcal{A}$,

$$
W^{*} A W\left(P_{1} H \otimes P_{2} H\right)=W^{*} A\left(P_{2} H \otimes P_{1} H\right) \subseteq W^{*}\left(P_{2} H \otimes P_{1} H\right)=P_{1} H \otimes P_{2} H .
$$

Consequently, $W^{*} \mathcal{A} W \subseteq \mathcal{A}$. Since $W=W^{*}$, it follows that $W \in N(\mathcal{A})$.

On the other hand, $W \notin N(\mathcal{B})$. In fact, let $P_{1}$ and $P_{2}$ be any nontrivial projections in $\mathcal{N}_{v}$ and let $V$ be a partial isometry with initial space $P_{1} H$ and final space $P_{1}^{\perp} H$. Then $V$ lies in $\mathcal{A}_{v}^{*}$. However,

$$
\begin{aligned}
W^{*}(I \otimes V) W\left(P_{1} H \otimes P_{2}^{\perp} H\right) & =W^{*}(I \otimes V)\left(P_{2}^{\perp} H \otimes P_{1} H\right) \\
& =W^{*}\left(P_{2}^{\perp} H \otimes P_{1}^{\perp} H\right)=P_{1}^{\perp} H \otimes P_{2}^{\perp} H,
\end{aligned}
$$

which shows that $W^{*}(I \otimes V) W \notin \mathcal{B}$. 
Now suppose that $n>2$. If $\mathcal{A} \neq \mathcal{B}$ and $\mathcal{A}^{*} \neq \mathcal{B}$ then there exist finite (possibly empty) tensor products $\mathcal{A}_{1}$ and $\mathcal{A}_{2}$ of copies of the Volterra nest algebra such that, up to a permutation of the factors in the tensor products,

$$
\mathcal{A}=\left(\mathcal{A}_{v} \otimes \mathcal{A}_{v}\right) \otimes \mathcal{A}_{1} \otimes \mathcal{A}_{2} \text { and } \mathcal{B}=\left(\mathcal{A}_{v} \otimes \mathcal{A}_{v}^{*}\right) \otimes \mathcal{A}_{1} \otimes \mathcal{A}_{2}^{*}
$$

Reasoning similar to that above shows that the operator $T=W \otimes I \otimes I$ lies in $N(\mathcal{A})$. If, additionally, $T \in N(\mathcal{B})$, then, by Lemma 4.7, the operator $W$ lies in $N\left(\mathcal{A}_{v} \otimes \mathcal{A}_{v}^{*}\right)$, yielding a contradiction.

Lemma 4.10. Let $(X, \mu)$ and $(Y, \nu)$ be standard measure spaces, let $\lambda \subseteq X \times X$ be a measurable subset, and let

$$
\kappa=\left\{\left(x_{1}, t, x_{2}, t\right):\left(x_{1}, x_{2}\right) \in \lambda, t \in Y\right\} .
$$

Then:

(i) $\lambda$ is marginally null if and only if $\kappa$ is marginally null in $(X \times Y) \times(X \times Y)$;

(ii) $\lambda$ is $\omega$-closed if and only if $\kappa$ is $\omega$-closed in $(X \times Y) \times(X \times Y)$.

Proof. For a subset $A \subseteq X \times Y$ and $t \in Y$, let $A^{t}=\{x \in X:(x, t) \in A\}$. If $A$ belongs to the product $\sigma$-algebra of $X \times Y$, we have that $A^{t}$ is measurable for all $t \in Y$.

(i) Suppose that $\lambda \subseteq(M \times X) \cup(X \times M)$ for some null set $M \subseteq X$. Then

$$
\kappa \subseteq((M \times Y) \times(X \times Y)) \cup((X \times Y) \times(M \times Y))
$$

is a marginally null set in $(X \times Y) \times(X \times Y)$.

Conversely, suppose that $\kappa \subseteq(N \times(X \times Y)) \cup((X \times Y) \times N)$, for some null set $N \subseteq X \times Y$. Since $N$ is null, we have that $N^{t}$ is null for almost all $t \in Y$. Pick such a $t$. Since $\lambda \subseteq\left(N^{t} \times X\right) \cup\left(X \times N^{t}\right)$, we have that $\lambda$ is marginally null.

(ii) Suppose that $\kappa^{c}$ is marginally equivalent to a subset of the form $E=$ $\cup_{i=1}^{\infty} A_{i} \times B_{i}$, where $A_{i}, B_{i} \subseteq X \times Y$ are measurable. There exists a null set $N \subseteq X \times Y$ such that

$$
(\kappa \cap E) \cup\left(\kappa^{c} \cap E^{c}\right) \subseteq(N \times(X \times Y)) \cup((X \times Y) \times N) .
$$

Fix $t \in Y$ and let $E(t)=\cup_{i=1}^{\infty} A_{i}^{t} \times B_{i}^{t}$. We claim that

$$
(\lambda \cap E(t)) \cup\left(\lambda^{c} \cap E(t)^{c}\right) \subseteq\left(N^{t} \times X\right) \cup\left(X \times N^{t}\right) .
$$

Suppose that $(x, y) \in \lambda \cap E(t)$. Then $(x, t, y, t) \in \kappa \cap E$ and hence either $(x, t) \in N$, in which case $x \in N^{t}$, or $(y, t) \in N$, in which case $y \in N^{t}$. Suppose, on the other hand, that $(x, y) \in \lambda^{c} \cap E(t)^{c}$. Then $(x, t, y, t) \in \kappa^{c} \cap E^{c}$; indeed, if $(x, t, y, t) \in E$ then $(x, y) \in A_{i}^{t} \times B_{i}^{t}$ for some $i$ and hence $(x, y) \in E(t)$, contrary to our assumption. It follows that $(x, t, y, t) \in(N \times(X \times Y)) \cup((X \times Y) \times N)$, and as before $(x, y) \in\left(N^{t} \times X\right) \cup\left(X \times N^{t}\right)$. It follows that $\lambda$ is $\omega$-closed.

For the converse direction, suppose that $\lambda^{c}$ is marginally equivalent to a subset of the form $\Omega=\cup_{i=1}^{\infty} \alpha_{i} \times \beta_{i}$, where $\alpha_{i}$ and $\beta_{i}$ are measurable, $i \in \mathbb{N}$, and let $M \subseteq X$ be a null set with

$$
(\lambda \cap \Omega) \cup\left(\lambda^{c} \cap \Omega^{c}\right) \subseteq(M \times X) \cup(X \times M) .
$$


Since $\kappa$ is the intersection of the sets $\kappa_{1}=\{(x, s, y, t):(x, y) \in \lambda, s, t \in Y\}$ and $\kappa_{2}=\{(x, t, y, t): x, y \in X, t \in Y\}$, it suffices to prove that both $\kappa_{1}$ and $\kappa_{2}$ are $\omega$-closed. However, $\kappa_{2}^{c}=\cup_{j=1}^{\infty}\left(X \times \gamma_{j}\right) \times\left(X \times \gamma_{j}^{c}\right)$, where $\left\{\gamma_{j}\right\}_{j \in \mathbb{N}}$ is a countable family generating the $\sigma$-algebra of $Y$ and is hence $\omega$-closed. On the other hand, if $\tilde{\Omega}=\cup_{i=1}^{\infty}\left(\alpha_{i} \times Y\right) \times\left(\beta_{i} \times Y\right)$ then

$$
\left(\kappa_{1} \cap \tilde{\Omega}\right) \cup\left(\kappa_{1}^{c} \cap \tilde{\Omega}^{c}\right) \subseteq(M \times Y \times X \times Y) \cup(X \times Y \times M \times Y)
$$

which shows that $\kappa_{1}$ is $\omega$-closed.

We are now ready to prove the main result of the present section.

Theorem 4.11. For all $i=1, \ldots, n$, let $H_{i}$ be a Hilbert space and let $\mathcal{A}_{i}$ be a continuous nest algebra acting on $H_{i}$. Let $\mathcal{H}=H_{1} \otimes \cdots \otimes H_{n}$, let $\mathcal{A}=\mathcal{A}_{1} \otimes \cdots \otimes \mathcal{A}_{n}$ and let $\mathcal{B}$ be a CSL algebra contained in $\mathcal{B}(\mathcal{H})$. Then $N(\mathcal{A})=N(\mathcal{B})$ if and only if $\mathcal{A}=\mathcal{B}$ or $\mathcal{A}^{*}=\mathcal{B}$.

Proof. As in the proof of Proposition 4.4 in [28], we may assume that $\mathcal{A} \cap \mathcal{A}^{*}$ is a masa, i.e., $\mathcal{A}=\operatorname{Alg}\left(\mathcal{N}_{1} \otimes \cdots \otimes \mathcal{N}_{n}\right)$ for some continuous multiplicity free nests $\mathcal{N}_{1}, \ldots, \mathcal{N}_{n}$ acting on $H_{1}, \ldots, H_{n}$, respectively. Suppose that the statement holds if $\mathcal{A}_{i}=\mathcal{A}_{v}$, for each $i=1, \ldots, n$. There exists a unitary operator $U_{i}: H \rightarrow H_{i}$ such that $U_{i} \mathcal{A}_{v} U_{i}^{*}=\mathcal{A}_{i}, i=1, \ldots, n$. Let $U=U_{1} \otimes \cdots \otimes U_{n}$, let $\mathcal{B}_{0}=U^{*} \mathcal{B} U$ and let $\mathcal{A}_{0}=\underbrace{\mathcal{A}_{v} \otimes \cdots \otimes \mathcal{A}_{v}}_{n}$. We have that $U^{*} \mathcal{A} U=\mathcal{A}_{0}$, and it is easy to see that the condition $N(\mathcal{A})=N(\mathcal{B})$ implies $N\left(\mathcal{A}_{0}\right)=N\left(\mathcal{B}_{0}\right)$. By assumption, $\mathcal{A}_{0}=\mathcal{B}_{0}$ or $\mathcal{A}_{0}=\mathcal{B}_{0}^{*}$, and hence $\mathcal{A}=\mathcal{B}$ or $\mathcal{A}=\mathcal{B}^{*}$. Hence, it suffices to establish the statement in the case $\mathcal{A}_{i}=\mathcal{A}_{v}$ for each $i=1, \ldots, n$.

We identify $\mathcal{H}$ with $L^{2}\left(\mathbb{I}^{n}\right)$. By Lemma 4.1 in $[28], N(\mathcal{A})=N(\mathcal{B})$ implies that $\mathcal{A} \cap \mathcal{A}^{*}=\mathcal{B} \cap \mathcal{B}^{*}$. We let $\kappa=\operatorname{supp} \mathcal{B} \subseteq \mathbb{I}^{n} \times \mathbb{I}^{n}$; note that $\kappa$ is an $\omega$-closed set.

Recall that $\Delta_{+}=\{(x, y) \in \mathbb{I} \times \mathbb{I}: x<y\}$ and let $\Delta_{-}=\{(x, y) \in \mathbb{I} \times \mathbb{I}: x>y\}$ and $\delta=\{(x, x): x \in \mathbb{I}\}$. Let $\rho: \mathbb{I}^{n} \times \mathbb{I}^{n} \rightarrow(\mathbb{I} \times \mathbb{I})^{n}$ be the mapping given by

$$
\rho\left(\left(x_{1}, \ldots, x_{n}, y_{1}, \ldots, y_{n}\right)\right)=\left(\left(x_{1}, y_{1}\right), \ldots,\left(x_{n}, y_{n}\right)\right) .
$$

For subsets $F \subseteq G \subseteq\{1, \ldots, n\}$, let $\Gamma_{F, G}=\lambda_{1} \times \cdots \times \lambda_{n} \subseteq(\mathbb{I} \times \mathbb{I})^{n}$, where

$$
\lambda_{i}= \begin{cases}\Delta_{+} & \text {if } i \in F, \\ \Delta_{-} & \text {if } i \in G \backslash F, \\ \delta & \text { if } i \notin G .\end{cases}
$$

Let also $\mathcal{E}_{F, G}=\mathcal{E}_{1} \otimes \cdots \otimes \mathcal{E}_{n}$, where

$$
\mathcal{E}_{i}= \begin{cases}\mathcal{A}_{v} & \text { if } i \in F, \\ \mathcal{A}_{v}^{*} & \text { if } i \in G \backslash F, \\ \mathcal{D} & \text { if } i \notin G .\end{cases}
$$

By Corollary 3.5, supp $\mathcal{E}_{F, G}=\rho^{-1}\left(\overline{\Gamma_{F, G}}\right)$ (here, and for the rest of this proof, $\bar{M}$ denotes the topological closure of the set $M)$. Set $\tilde{\delta}=\Gamma_{\emptyset, \emptyset}$; clearly, $\rho_{n}^{-1}(\tilde{\delta})$ is the support of the masa $\underbrace{\mathcal{D} \otimes \cdots \otimes \mathcal{D}}_{n}$. 
If $F \subseteq G$ are as above and $k$ is the cardinality of $G$, let $\Delta_{F, G}=\lambda_{1} \times \cdots \times \lambda_{k}$ where

$$
\lambda_{i}= \begin{cases}\Delta_{+} & \text {if } i \in F, \\ \Delta_{-} & \text {if } i \in G \backslash F\end{cases}
$$

thus, $\Delta_{F, G} \subseteq(\mathbb{I} \times \mathbb{I})^{k}$. Let $\mathcal{C}_{F, G}=\mathcal{C}_{1} \otimes \cdots \otimes \mathcal{C}_{k}$ where

$$
\mathcal{C}_{i}= \begin{cases}\mathcal{A}_{v} & \text { if } i \in F \\ \mathcal{A}_{v}^{*} & \text { if } i \in G \backslash F .\end{cases}
$$

We have that

$$
(\mathbb{I} \times \mathbb{I})^{n}=\bigcup\left\{\Gamma_{F, G}: F \subseteq G \subseteq\{1, \ldots, n\}\right\} .
$$

We establish the following

Claim. Suppose that $\kappa \cap \rho^{-1}\left(\Gamma_{F, G}\right) \neq \emptyset$ for some $F \subseteq G \subseteq\{1, \ldots, n\}$. Then $\mathcal{E}_{F, G} \subseteq \mathcal{B}$.

Proof of the claim. It is clear that $\Gamma_{\emptyset, \emptyset}=\tilde{\delta}$, so $\mathcal{E}_{\emptyset, \emptyset}=\underbrace{\mathcal{D} \otimes \cdots \otimes \mathcal{D}}_{n}$. Since $\mathcal{B} \cap \mathcal{B}^{*}=$ $\mathcal{E}_{\emptyset, \emptyset}$, the claim is proved in the case $F=G=\emptyset$.

Suppose that $G \neq \emptyset$. We may assume, after permuting the terms if necessary, that there exist $k$ and $l \leq k$ such that $G=\{1, \ldots, k\}$ and, if $F \neq \emptyset$, then $F=$ $\{1, \ldots, l\}$. Let $\kappa_{0}=\left(\kappa \cap \rho^{-1}\left(\overline{\Gamma_{F, G}}\right)\right) \subseteq \mathbb{I}^{n} \times \mathbb{I}^{n}$. Then there exists $\lambda \subseteq \overline{\Delta_{F, G}}$ such that $\kappa_{0}=\rho_{n}^{-1}(\lambda \times \underbrace{\delta \times \cdots \times \delta}_{n-k \text { times }})$. By Corollary 3.5, we have that

$$
\operatorname{supp}(\mathcal{M}_{\max }\left(\rho_{k}^{-1}(\lambda)\right) \otimes \underbrace{\mathcal{D} \otimes \cdots \otimes \mathcal{D}}_{n-k}) \subseteq \kappa .
$$

Since $\mathcal{B}$ is reflexive, it follows that

$$
\mathcal{M}_{\max }\left(\rho_{k}^{-1}(\lambda)\right) \otimes \underbrace{\mathcal{D} \otimes \cdots \otimes \mathcal{D}}_{n-k} \subseteq \mathcal{B} .
$$

We claim that $\rho_{k}^{-1}\left(\lambda \cap \Delta_{F, G}\right) \not \emptyset$. To see this, suppose that $\rho_{k}^{-1}\left(\lambda \cap \Delta_{F, G}\right) \simeq \emptyset$. By Lemma 4.10 (i), we have that

$$
\kappa \cap \rho^{-1}\left(\Gamma_{F, G}\right)=\rho_{2}^{-1}\left(\rho_{k}^{-1}\left(\lambda \cap \Delta_{F, G}\right) \times \rho_{n-k}^{-1}(\delta \times \cdots \times \delta)\right)
$$

is marginally null, a contradiction. By Lemma 4.10 (ii), it follows that $\rho_{k}^{-1}(\lambda \cap$ $\overline{\Delta_{F, G}}$ ) is $\omega$-closed. Lemma 4.4 and the fact that every continuous multiplicity free nest is unitarily equivalent to the Volterra nest imply that there exist $P_{i} \in \operatorname{Lat} \mathcal{C}_{i}$, $i=1, \ldots, k$, and a non-zero operator $A$ supported on $\lambda$ such that

$$
A=\left(P_{1} \otimes \cdots \otimes P_{k}\right) A\left(P_{1}^{\perp} \otimes \cdots \otimes P_{k}^{\perp}\right) .
$$

By (4.4), letting $I_{n-k}$ denote the identity operator on $H_{k+1} \otimes \cdots \otimes H_{n}$, it follows that $A \otimes I_{n-k} \in \mathcal{B}$. We claim that $A \in \mathcal{C}_{F, G}$. To show this, let $L_{i} \in \operatorname{Lat} \mathcal{C}_{i}$, $i=1, \ldots, k$. If $L_{i} \leq P_{i}$ for some $i$ then

$$
\left(L_{1} \otimes \cdots \otimes L_{k}\right)\left(P_{1}^{\perp} \otimes \cdots \otimes P_{k}^{\perp}\right)=0 .
$$


If $P_{i} \leq L_{i}$ for each $i$ then $P_{1} \otimes \cdots \otimes P_{k} \leq L_{1} \otimes \cdots \otimes L_{k}$ and hence

$$
\left(P_{1} \otimes \cdots \otimes P_{k}\right)\left(L_{1} \otimes \cdots \otimes L_{k}\right)^{\perp}=0 .
$$

Thus, in both cases, $\left(L_{1} \otimes \cdots \otimes L_{k}\right)^{\perp} A\left(L_{1} \otimes \cdots \otimes L_{k}\right)=0$ and hence $A \in \mathcal{C}_{F, G}$.

By Theorem 4.6,

$$
\left.\mathcal{C}_{F, G}=\overline{\left[(S T) A(S T)^{*}: S \in N_{e}\left(\mathcal{C}_{F, G}\right), T \in N_{P}\left(\mathcal{C}_{F, G}\right)\right.}\right]^{w^{*}},
$$

where $P=P_{1} \otimes \cdots \otimes P_{k}$. Let $\mathcal{A}_{k}^{0}=\underbrace{\mathcal{A}_{v} \otimes \cdots \otimes \mathcal{A}_{v}}_{k}$. Using Lemma 4.2 and the fact that $N_{e}\left(\mathcal{A}_{k}^{0}\right)=N_{e}\left(\mathcal{C}_{F, G}\right)$, we conclude that

$$
\begin{aligned}
\mathcal{C}_{F, G} \otimes I_{n-k} & \subseteq \overline{\left[\left(V \otimes I_{n-k}\right)\left(A \otimes I_{n-k}\right)\left(V \otimes I_{n-k}\right)^{*}: V \in N\left(\mathcal{A}_{k}^{0}\right)\right]} w^{*} \\
& \subseteq \overline{\left[W\left(A \otimes I_{n-k}\right) W^{*}: W \in N(\mathcal{B})\right]} w^{*} \subseteq \mathcal{B} .
\end{aligned}
$$

Since $\underbrace{\mathcal{D} \otimes \cdots \otimes \mathcal{D}}_{n} \subseteq \mathcal{B}$, this implies that $\mathcal{E}_{F, G} \subseteq \mathcal{B}$. The claim is proved.

We now finish the proof of the theorem. Suppose that $\kappa \cap \rho^{-1}\left(\Gamma_{F_{1}, G_{1}}\right)$ and $\kappa \cap \rho^{-1}\left(\Gamma_{F_{2}, G_{2}}\right)$, where $F_{1} \subseteq G_{1} \subseteq\{1, \ldots, n\}$ and $F_{2} \subseteq G_{2} \subseteq\{1, \ldots, n\}$, are both not marginally null. Suppose, moreover, that there exists

$$
i \in\left(F_{2} \cap\left(G_{1} \backslash F_{1}\right)\right) \bigcup\left(F_{1} \cap\left(G_{2} \backslash F_{2}\right)\right) .
$$

Let $\mathcal{F}_{1}$ (respectively, $\mathcal{F}_{2}$ ) denote the tensor product of length $n$ whose $j$ th term is equal to $\mathcal{D}$ if $j \neq i$ and to $\mathcal{A}_{v}$ (respectively, $\mathcal{A}_{v}^{*}$ ) if $j=i$. From the Claim, we have that $\mathcal{F}_{1}+\mathcal{F}_{2} \subseteq \mathcal{B}$. Let $\mathcal{F}_{3}$ denote the tensor product of length $n$ whose $j$ th term is equal to $\mathcal{D}$ if $j \neq i$ and to $\mathcal{B}(H)$ if $j=i$. By Corollary 4.2.5 in [19], $\overline{\mathcal{A}}_{v}+\mathcal{A}_{v}^{*}{ }^{w *}=\mathcal{B}(H)$ and it follows that $\mathcal{F}_{3} \subseteq \mathcal{B}$. However, this contradicts the fact that the diagonal of $\mathcal{B}$ is a masa.

It follows that if $\kappa$ intersects in a non-marginally null set the subsets $\rho^{-1}\left(\Gamma_{F_{1}, G_{1}}\right)$ and $\rho^{-1}\left(\Gamma_{F_{2}, G_{2}}\right)$, where $F_{1} \subseteq G_{1} \subseteq\{1, \ldots, n\}$ and $F_{2} \subseteq G_{2} \subseteq\{1, \ldots, n\}$, then

$$
\left(F_{2} \cap\left(G_{1} \backslash F_{1}\right)\right)=\emptyset \text { and }\left(F_{1} \cap\left(G_{2} \backslash F_{2}\right)\right)=\emptyset \text {. }
$$

Thus,

$$
\left(F_{1} \cup F_{2}\right) \cap\left(\left(G_{1} \backslash F_{1}\right) \cup\left(G_{2} \backslash F_{2}\right)\right)=\emptyset
$$

and hence $\overline{\Gamma_{F_{1}, G_{1}}} \cup \overline{\Gamma_{F_{2}, G_{2}}}=\overline{\Gamma_{F_{3}, G_{3}}}$, where $F_{3}=F_{1} \cup F_{2}$ and $G_{3}=G_{1} \cup G_{2}$. Therefore, there exist $F \subseteq G \subseteq\{1, \ldots, n\}$ such that if $\kappa \cap \rho^{-1}\left(\Gamma_{J, K}\right) \not 千 \emptyset$ then $\overline{\Gamma_{J, K}} \subseteq \overline{\Gamma_{F, G}}$.

Since $\kappa$ is non-marginally null, (4.3) implies that there exist $J \subseteq K \subseteq\{1, \ldots, n\}$ such that $\kappa \cap \rho^{-1}\left(\Gamma_{J, K}\right) \not \nsucc$. By the Claim and Corollary 3.5, $\rho^{-1}\left(\overline{\Gamma_{J, K}}\right) \subseteq \kappa$. It follows that there exist $F \subseteq G \subseteq\{1, \ldots, n\}$ such that $\rho^{-1}\left(\overline{\Gamma_{F, G}}\right)=\kappa$ and hence $\kappa=\operatorname{supp} \mathcal{E}_{F, G}$. Therefore, $\mathcal{B}=\mathcal{E}_{F, G}$. If $G \neq\{1, \ldots, n\}$ then Lemma 4.8 would imply that $N(\mathcal{D})=N\left(\mathcal{A}_{v}\right)$, a contradiction. Hence $G=\{1, \ldots, n\}$. Lemma 4.9 now implies that either $\mathcal{A}=\mathcal{B}$ or $\mathcal{A}^{*}=\mathcal{B}$. 
We include an immediate consequence of Theorem 4.11, generalising Proposition 4.4 in [28].

Corollary 4.12. Let $\mathcal{H}$ be a Hilbert space, let $\mathcal{A}$ be a continuous nest algebra acting on $\mathcal{H}$ and let $\mathcal{B}$ be a CSL algebra contained in $\mathcal{B}(\mathcal{H})$. If $N(\mathcal{A})=N(\mathcal{B})$, then either $\mathcal{A}=\mathcal{B}$ or $\mathcal{A}^{*}=\mathcal{B}$.

\section{An example}

In this section, we give an example which shows that Theorem 4.11 does not hold without the assumption that the nest algebras $\mathcal{A}_{i}$ be continuous. This example settles, simultaneously, question (1.1) in the negative.

We will need some facts from [2] and [28] which we now recall. Let $(X, \mu)$ be a standard measure space. A quasi-order $\leq$ on $X$ is called standard if there exist measurable functions $f_{n}: X \rightarrow \mathbb{R}, n \in \mathbb{N}$, such that $x \leq y$ if and only if $f_{n}(x) \leq f_{n}(y)$ for all $n \in \mathbb{N}$. If $(Y, \preceq, \nu)$ is another standard quasi-ordered measure space, a function $f: X_{0} \rightarrow Y$ (where $X_{0} \subseteq X$ is measurable) is called almost increasing if there exists a null set $N \subseteq X$ such that $x, y \in X_{0} \backslash N, x \leq y$ imply that $f(x) \preceq f(y)$. A subset $E \subseteq X$ is called almost increasing if $\chi_{E}: X \rightarrow \mathbb{R}$ is an almost increasing function, where $\mathbb{R}$ is equipped with its standard order.

Let $\mathcal{L}(X, \leq, \mu)$ be the collection of all projections acting on $L^{2}(X, \mu)$ that are multiplication by characteristic functions of almost increasing sets; then $\mathcal{L}(X, \leq, \mu)$ is a CSL. Arveson showed [2] that every separably acting CSL is unitarily equivalent to $\mathcal{L}(X, \leq, \mu)$ for some standard quasi-ordered measure space $(X, \leq, \mu)$. It was shown in [13] that $\mathcal{L}(X, \leq, \mu) \otimes \mathcal{L}(Y, \preceq, \nu)$ is unitarily equivalent to $\mathcal{L}(X \times Y, \leq$ $\times \preceq, \mu \times \nu)$, where $\leq \times \preceq$ is the product order on $X \times Y$.

Let $(X, \leq, \mu)$ and $(Y, \preceq, \nu)$ be standard quasi-ordered measure spaces. A measurable mapping $f: X_{0} \rightarrow Y$, defined on a measurable subset $X_{0}$ of $X$, is called a partial Borel isomorphism if

(i) there exists a null set $N \subseteq X_{0}$ such that the restriction of $f$ to $X_{0} \backslash N$ is injective;

(ii) the inverse mapping $f^{-1}: f\left(X_{0} \backslash N\right) \rightarrow X_{0}$ is measurable, and

(iii) $f$ and $f^{-1}$ are null set preserving.

If, furthermore, $N$ can be chosen such that for all $x$ and $z$ in $X_{0} \backslash N$,

(iv) $x \leq z \Longleftrightarrow f(x) \preceq f(z)$,

then $f$ is said to be a partial order isomorphism. It was shown in [28] that, if $\leq$ is a partial order (equivalently, if $\mathcal{L}(X, \leq, \mu)$ is multiplicity free), then an operator $T \in \mathcal{B}\left(L^{2}(X, \mu)\right)$ is a normaliser of $\operatorname{Alg} \mathcal{L}(X, \leq, \mu)$ if and only if $T$ is supported on a set of the form $\left\{(x, f(x)): x \in X_{0}\right\}$, where $f: X_{0} \rightarrow X$ is a partial order isomorphism. Consequently, two multiplicity free CSL algebras $\operatorname{Alg} \mathcal{L}(X, \leq, \mu)$ and $\operatorname{Alg} \mathcal{L}(X, \preceq, \mu)$ have the same normaliser semigroups if and only if the partial orders $\leq$ and $\preceq$ have the same partial order isomorphisms. 
Let $H_{0}$ be a complex Hilbert space, let $E$ be the identity on $H_{0}$, let $\mathcal{N}_{v}$ be the Volterra nest acting on $H=L^{2}(\mathbb{I})$, and let

$$
\mathcal{N}_{1}=\left\{0 \oplus N_{t}: 0 \leq t \leq 1\right\} \cup\{I\} \quad \text { and } \quad \mathcal{N}_{2}=\{0\} \cup\left\{E \oplus N_{t}: 0 \leq t \leq 1\right\} ;
$$

note that $\mathcal{N}_{1}$ and $\mathcal{N}_{2}$ are nests acting on $H_{0} \oplus H$.

Let $X=(\{p\} \cup \mathbb{I}) \times \mathbb{I}$, equipped with the product measure $\mu$, where the space $\{p\} \cup \mathbb{I}$ is the disjoint sum of the counting measure on $\{p\}$ and Lebesgue measure on $\mathbb{I}$ (here $p$ is an arbitrary element not belonging to $\mathbb{I}$ ). Equip $\{p\} \cup \mathbb{I}$ with the total orders $\preceq_{1}$ and $\preceq_{2}$ both of which coincide with the usual order on $\mathbb{I}$, and $p \preceq_{1} t$ (resp. $t \preceq_{2} p$ ) for all $t \in \mathbb{I}$. Let $\leq_{1}\left(\right.$ resp. $\leq_{2}$ ) be the product order on $X$, where $\{p\} \cup \mathbb{I}$ is equipped with $\preceq_{1}$ (resp. $\preceq_{2}$ ), while $\mathbb{I}$ is equipped with the usual order. It follows from [13] that $\mathcal{N}_{1} \otimes \mathcal{N}_{v}=\mathcal{L}\left(X, \leq_{1}, \mu\right)$ and $\mathcal{N}_{2} \otimes \mathcal{N}_{v}=\mathcal{L}\left(X, \leq_{2}, \mu\right)$. Let $\mathcal{A}_{1}=\operatorname{Alg}\left(\mathcal{N}_{1} \otimes \mathcal{N}_{v}\right)$ and $\mathcal{A}_{2}=\operatorname{Alg}\left(\mathcal{N}_{2} \otimes \mathcal{N}_{v}\right)$.

Lemma 5.1. Let $f: X_{0} \rightarrow X$ be a partial order isomorphism of $\left(X, \leq_{1}, \mu\right)$ or of $\left(X, \leq_{2}, \mu\right)$. Then there exists a set $\Lambda \subseteq \mathbb{I}$ of full Lebesgue measure such that the set $X_{0} \cap(\{p\} \times \Lambda)$ is mapped into $\{p\} \times \mathbb{I}$.

Proof. Let $N \subseteq X$ be a null set such that conditions (i)-(iv) from the definition of a partial order isomorphism hold, and let $N_{p} \subseteq \mathbb{I}$ be the set with $N \cap(\{p\} \times \mathbb{I})=$ $\{p\} \times N_{p}$; clearly, $N_{p}$ is null. Further, let $\mathrm{M} \subseteq \mathbb{I}$ be the (measurable) subset with the property that $\left(X_{0} \backslash N\right) \cap(\{p\} \times \mathbb{I})=\{p\} \times \mathrm{M}$. We may assume that $\mathrm{M}$ is not null for otherwise we can set $\Lambda=\left(\mathrm{M} \cup N_{p}\right)^{c}$. Let $\mathrm{J}=f(\{p\} \times \mathrm{M}) \cap(\mathbb{I} \times \mathbb{I})$. For each $x \in \mathbb{I}$, let $\mathrm{J}_{x}=\{y:(x, y) \in \mathrm{J}\} \subseteq \mathbb{I}$ be the vertical section of $\mathrm{J}$ corresponding to $x$. Let $Q=\left\{x \in \mathbb{I}: \mathrm{J}_{x}\right.$ is not null $\}$. Since $f^{-1}$ is null set preserving and strictly increasing on $\mathrm{J}$ (when the latter is equipped with the product order), we have that $\left\{f^{-1}\left(\mathrm{~J}_{x}\right): x \in Q\right\}$ is a family of non-null pairwise disjoint subsets of $\mathbb{I}$; hence, $Q$ is countable. It follows that $\mathrm{J}_{x}$ is null for almost all $x$; this means that $\mathrm{J}$ is null. Let $R \subseteq \mathbb{I}$ be the (measurable) subset such that $\{p\} \times R=f^{-1}(\mathrm{~J})$; then $R$ is null since $f^{-1}$ is null set preserving. To complete the proof, let $\Lambda=\mathbb{I} \backslash\left(R \cup N_{p}\right)$.

Let $X_{0} \subseteq X$ be a measurable set and $f: X_{0} \rightarrow X$ be a partial Borel isomorphism. Let $f_{1}: X_{0} \rightarrow\{p\} \cup \mathbb{I}$ and $f_{2}: X_{0} \rightarrow \mathbb{I}$ be the functions given by the identity $f(x, y)=\left(f_{1}(x, y), f_{2}(x, y)\right),(x, y) \in X_{0}$. Clearly, $f_{1}$ and $f_{2}$ are measurable.

Let $f$ be a partial $\leq_{1}$-order isomorphism (respectively, a partial $\leq_{2}$-order isomorphism) and let $N \subseteq X$. For all $t \in\left\{s: \exists x\right.$ with $\left.(x, s) \in X_{0} \backslash N\right\}$, let $\Omega_{t}^{N}$ be the open (possibly empty) interval defined by

$$
\Omega_{t}^{N}=\left(\inf _{(x, t) \in X_{0} \backslash N} f_{2}(x, t), \sup _{(x, t) \in X_{0} \backslash N} f_{2}(x, t)\right) .
$$

Lemma 5.2. Let $f: X_{0} \rightarrow X$ be a partial order isomorphism of $\left(X, \leq_{1}, \mu\right)$ (respectively, of $\left.\left(X, \leq_{2}, \mu\right)\right)$. Let $\Lambda \subseteq \mathbb{I}$ be the set from Lemma 5.1 and let $N \subseteq X$ be a null set containing $\{p\} \times \Lambda^{c}$ which satisfies conditions (i)-(iv) from the definition of a partial order isomorphism with respect to $\leq_{1}$ (respectively, $\leq_{2}$ ). If $t$ and $s$ are distinct elements lying in the set $\left\{r:(p, r) \in X_{0} \backslash N\right\}$, then $\Omega_{s}^{N} \cap \Omega_{t}^{N}=\emptyset$. 
Proof. Set $\Omega_{s}=\Omega_{s}^{N}$ and $\Omega_{t}=\Omega_{t}^{N}$, and suppose that $s<t$ while $\Omega_{s} \cap \Omega_{t} \neq \emptyset$. Assume first that $f$ is a partial order isomorphism of $\left(X, \leq_{1}, \mu\right)$. We have that

$$
\Omega_{t}=\left(f_{2}(p, t), \sup _{(x, t) \in X_{0} \backslash N} f_{2}(x, t)\right) .
$$

Then there exists $(a, s) \in X_{0} \backslash N$ such that $f_{2}(a, s)>f_{2}(p, t)$. Since $f_{1}(p, t)=p$ (recall the definition of $N)$, we have that $f(p, t) \leq_{1} f(a, s)$. Thus, $(p, t) \leq(a, s)$, which implies that $t \leq s$, a contradiction.

Now suppose that $f$ is a partial order isomorphism of $\left(X, \leq_{2}, \mu\right)$. Then

$$
\Omega_{s}=\left(\inf _{(x, s) \in X_{0} \backslash N} f_{2}(x, s), f_{2}(p, s)\right) .
$$

Then there exists $(a, t) \in X_{0} \backslash N$ such that $f(a, t) \leq_{2} f(p, s)$ with $(a, t) \mathbb{L}_{2}(p, s)$, contradicting the fact that, for all $(x, y) \in X_{0} \backslash N$,

$$
(x, y) \leq_{2}(z, w) \Leftarrow f(x, y) \leq_{2} f(z, w) .
$$

Proposition 5.3. Let $f: X_{0} \rightarrow X$ be a partial Borel isomorphism defined on a subset $X_{0}$ of $X$. Then, $f$ is a partial order isomorphism of $\left(X, \leq_{1}, \mu\right)$ if and only if $f$ is a partial order isomorphism of $\left(X, \leq_{2}, \mu\right)$.

Proof. Suppose that $f$ is a partial order isomorphism of $\left(X, \leq_{1}, \mu\right)$. By the definition of partial order isomorphism and Lemma 5.1, there exists a null subset $N$ of the set $X$ such that, for all $(x, y)$ and $(z, w)$ in $X_{0} \backslash N$,

$$
(x, y) \leq_{1}(z, w) \Longleftrightarrow f(x, y) \leq_{1} f(z, w),
$$

$f\left(\left(X_{0} \backslash N\right) \cap(\{p\} \times \mathbb{I})\right) \subseteq\{p\} \times \mathbb{I}$ and $f\left(\left(X_{0} \backslash N\right) \cap(\mathbb{I} \times \mathbb{I})\right) \subseteq \mathbb{I} \times \mathbb{I}$. (The last inclusion can be ensured by considering $f^{-1}$ in place of $f$ in Lemma 5.1.) Since the partial orders $\leq_{1}$ and $\leq_{2}$ coincide on $\{p\} \times \mathbb{I}$ and on $\mathbb{I} \times \mathbb{I}$, we have that for all $(p, s),(p, t) \in X_{0} \backslash N$,

$$
(p, s) \leq_{2}(p, t) \Longleftrightarrow f(p, s) \leq_{2} f(p, t)
$$

and for all $(x, y),(z, w) \in\left(X_{0} \backslash N\right) \cap(\mathbb{I} \times \mathbb{I})$,

$$
(x, y) \leq_{2}(z, w) \Longleftrightarrow f(x, y) \leq_{2} f(z, w) .
$$

Define the sets $X_{a}$ and $X_{b}$, respectively, by

$$
\begin{aligned}
& X_{a}=\left\{(p, t) \in X_{0} \backslash N: \exists(x, y)\right. \in\left(X_{0} \backslash N\right) \cap(\mathbb{I} \times \mathbb{I}): \\
&(x, y) \leq_{2}(p, t) \text { and } f(x, y) \\
&\left.\leq_{2} f(p, t)\right\}
\end{aligned}
$$

and

$$
\begin{aligned}
X_{b}=\left\{(p, t) \in X_{0} \backslash N: \exists(x, y)\right. & \in\left(X_{0} \backslash N\right) \cap(\mathbb{I} \times \mathbb{I}): \\
(x, y) & \left.\leq_{2}(p, t) \text { and } f(x, y) \leq_{2} f(p, t)\right\} .
\end{aligned}
$$


First, observe that $X_{b}=\emptyset$. In fact, if $(p, t)$ lies in $X_{b}$, then there exists $(x, y) \in$ $\left(X_{0} \backslash N\right) \cap(\mathbb{I} \times \mathbb{I})$ such that $t<y$ and $f(x, y) \leq_{2} f(p, t)$. Since $f(p, t)$ lies in $\{p\} \times \mathbb{I}$, we have that $f_{2}(x, y) \leq f_{2}(p, t)$. It follows that $(p, t) \leq_{1}(p, y) \leq_{1}(x, y)$ and, since $f$ is a partial order isomorphism of $\left(X, \leq_{1}, \mu\right)$, we have $f(p, t) \leq_{1} f(p, y) \leq_{1} f(x, y)$. Hence, by the definition of $\leq_{1}$,

$$
f_{2}(p, t) \leq f_{2}(p, y) \leq f_{2}(x, y)
$$

Since, as previously seen, $f_{2}(x, y) \leq f_{2}(p, t)$, it follows that $f_{2}(p, t)=f_{2}(p, y)$. Therefore $f(p, t)=f(p, y)$ and $(p, t) \neq(p, y)$, contradicting the fact that $f$ is injective on $X_{0} \backslash N$. Consequently, the set $X_{b}$ is empty.

Suppose now that $(p, t)$ is an element of $X_{a}$. In this case, $y \leq t$ and $f_{2}(p, t)<$ $f_{2}(x, y)$ (for if $f_{2}(x, y) \leq f_{2}(p, t)$ then, since $f_{1}(p, t)=p$, by the definition of the order $\leq_{2}$, we would have that $\left.f(x, y) \leq_{2} f(p, t)\right)$. It follows that $f(p, t) \leq_{1} f(x, y)$. Therefore, if $y<t$ then $(p, t) \underline{\leq}_{1}(x, y)$ and $f(p, t) \leq_{1} f(x, y)$, which contradicts the fact that $f$ satisfies condition (5.1) on $X_{0} \backslash N$. Consequently, if $(p, t)$ lies in $X_{a}$ and $(x, y)$ is an element of $\left(X_{0} \backslash N\right) \cap(\mathbb{I} \times \mathbb{I})$ satisfying the condition in the definition of the set $X_{a}$, then $y=t$.

Let $Z$ be the subset of $\mathbb{I}$ defined by

$$
Z=\left\{t \in \mathbb{I}:(p, t) \in X_{a}\right\} .
$$

Recall that, if $t \in Z$ then there exists $x \in \mathbb{I}$ such that $f_{2}(p, t)<f_{2}(x, t)$. It follows from Lemma 5.2 that the sets $\Omega_{t}^{N}, t \in Z$, are nontrivial pairwise disjoint open subintervals of $\mathbb{I}$; thus, $Z$ is countable. Consequently, $M=\mathbb{I} \times Z$ is a null set. Moreover, if $x, y \in X_{0} \backslash(N \cup M)$, then $x \leq_{2} y$ if and only if $f(x) \leq_{2} f(y)$. It follows that $f$ is a partial order isomorphism of $\left(X, \leq_{2}, \mu\right)$.

We have shown that every partial order isomorphism of $\left(X, \leq_{1}, \mu\right)$ is a partial order isomorphism of $\left(X, \leq_{2}, \mu\right)$. Similar reasoning shows that the converse is also true, and the proof is complete.

Corollary 5.4. The algebras $\mathcal{A}_{1}$ and $\mathcal{A}_{2}$ are continuous $C S L$ algebras such that $\mathcal{A}_{1} \neq \mathcal{A}_{2}, \mathcal{A}_{1}^{*} \neq \mathcal{A}_{2}$ and $N\left(\mathcal{A}_{1}\right)=N\left(\mathcal{A}_{2}\right)$.

Proof. By Theorem 2.2 in [28] and Proposition 5.3, $N\left(\mathcal{A}_{1}\right)=N\left(\mathcal{A}_{2}\right)$. The inequalities are straightforward.

\section{References}

[1] Anoussis, M. and Katavolos, A.: Unitary actions on nests and the Weyl relations. Bull. London Math. Soc. 27 (1995), no. 3, 265-272.

[2] Arveson, W.: Operator algebras and invariant subspaces. Ann. of Math. (2) 100 (1974), 433-532.

[3] Blecher, D. P. And Le Merdy, C.: Operator algebras and their modules - an operator space approach. London Mathematical Society Monographs, New Series 30, Oxford Science Publications. The Clarendon Press, Oxford University Press, Oxford, 2004. 
[4] Chifan, I.: On the normalizing algebra of a masa in a $I I_{1}$ factor. Preprint.

[5] Contes, K. J.: Normalizers of nest algebras. Proc. Amer. Math. Soc. 126 (1998), no. 1, 159-165; Errata, ibid, no. 8, 2511-2512.

[6] Davidson, K. R.: Nest algebras. Triangular forms for operator algebras on Hilbert space. Pitman Research Notes in Mathematics 191, Longman Scientific \& Technical, Harlow, 1988

[7] Davidson, K. R.: Normalizers of finite multipliciy nests. Proc. Edinburgh Math. Soc. (2) 39 (1996), no. 2, 337-344.

[8] Eleftherakis, G. K.: TRO equivalent algebras. Houston J. Math. 38 (2012), no. 1, $153-175$.

[9] Eleftherakis, G. K.: A Morita type equivalence for dual operator algebras. J. Pure Appl. Algebra 212 (2008), no. 5, 1060-1071.

[10] Eleftherakis, G. K. And Paulsen, V. I.: Stably isomorphic dual operator algebras. Math. Ann. 341 (2008), no. 1, 99-112.

[11] Erdos, J. A., Katavolos, A. and Shulman, V. S.: Rank one subspaces of bimodules over maximal abelian self-adjoint algebras. J. Funct. Anal. 157 (1998), no. 2, $554-587$.

[12] Fang, J., Smith, R. R., White, S. A. and Wiggins, A. D.: Groupoid normalizers of tensor products. J. Funct. Anal. 258 (2010), no. 1, 20-49.

[13] Gilfeather, F., Hopenwasser, A. and Larson, D. R.: Reflexive algebras with finite width lattices: tensor products, cohomology, compact perturbations. J. Funct. Anal. 55 (1984), no. 2, 176-199.

[14] Halmos, P. R.: Limsups of Lats. Indiana Univ. Math. J. 29 (1980), no. 2, 293-311.

[15] Hopenwasser, A.: Tensor products of reflexive subspace lattices. Michigan Math. J. 31 (1984), no. 3, 359-370.

[16] Kadison, R. V. And Ringrose, J. R.: Fundamentals of the theory of operator algebras. Vol. II. Advanced theory. Pure and Applied Mathematics 100, Academic Press, Orlando, FL, 1986.

[17] Katavolos, A. And Todorov ,I. G.: Normalizers of operator algebras and reflexivity. Proc. London Math. Soc. (3) 86 (2003), no. 2, 463-484.

[18] Kraus, J.: The slice map problem for $\sigma$-weakly closed subspaces of von Neumann algebras. Trans. Amer. Math. Soc. 279 (1983), no. 1, 357-376.

[19] Loebl, R. I. And Muhly, P. S.: Analyticity and flows in von Neumann algebras. J. Funct. Anal. 29 (1978), no. 2, 214-251.

[20] Loginov, A. I. And Shulman, V.S.: Hereditary and intermediate reflexivity of W*-algebras. Izv. Akad. Nauk. SSSR Ser. Mat. 39 (1975), no. 6, 1260-1273, 1437.

[21] McGarvey, M. And Todorov, I. G.: Normalisers, nest algebras and tensor products. In Operator structures and dynamical systems, 141-149. Contemp. Math. 503, Amer. Math. Soc., Providence, RI, 2009.

[22] Pisier, G.: Similarity problems and completely bounded maps. Lecture Notes in Mathematics 1618, Springer-Verlag, Berlin, 2001.

[23] Power, S. C.: Limit algebras: an introduction to subalgebras of $C^{*}$-algebras. Pitman Research Notes in Mathematics Series 278, Longman Scientific \& Technical, Harlow, 1992. 
[24] Shulman, V.S. And Todorov, I. G.: On subspace lattices I. Closedness type properties and tensor products. Integral Equations Operator Theory 52 (2005), no. 4, 561-579.

[25] Shulman, V.S. And Turowska, L.: Operator synthesis. I. Synthetic sets, bilattices and tensor algebras. J. Funct. Anal. 209 (2004), no. 2, 293-331.

[26] Sinclair, A. M., Smith, R. R., White, S. A. And Wiggins, A. D.: Strong singularity of singular masas in $I I_{1}$ factors. Illinois J. Math. 51 (2007), no. 4, 1077-1084.

[27] Smith, R. R., White, S. A. And Wiggins, A. D.: Normalizers of irreducible subfactors. J. Math. Anal. Appl. 352 (2009), no. 2, 684-695.

[28] Todorov, I. G.: Normalisers of CSL algebras. Indiana Univ. Math. J. 53 (2004), no. $4,1013-1036$.

[29] Todorov, I. G.: Subspace lattices, reflexivity and tensor products. Irish Math. Soc. Bull. 53 (2004), 57-67.

[30] Williams, D. P.: Crossed products of $C^{*}$-algebras. Mathematical Surveys and Monographs 134, American Mathematical Society, Providence, RI, 2007.

Received February 2, 2012.

Martin McGarvey: Pure Mathematics Research Centre, Queen's University Belfast, Belfast BT7 1NN, United Kingdom.

E-mail: mmcgarvey07@qub.ac.uk

Lina Oliveira: Center for Mathematical Analysis, Geometry and Dynamical Systems; and Department of Mathematics, Instituto Superior Técnico, Universidade de Lisboa, Av. Rovisco Pais, 1049-001 Lisboa, Portugal.

E-mail: linaoliv@math.ist.utl.pt

Ivan G. TOdorov: Pure Mathematics Research Centre, Queen's University Belfast, Belfast BT7 1NN, United Kingdom.

E-mail: i.todorov@qub.ac.uk

The first author was supported by a grant from the Department for Employment and Learning of Northern Ireland. The second author was partially supported by CAMGSD-LARSyS through the FCT Program POCTI-FEDER and by the FCT project EXCL/MAT-GEO/0222/2012. 